\title{
Challenges and opportunities in the delivery of cancer therapeutics: update on recent progress
}

\author{
Mathilde Lorscheider ${ }^{\star \ddagger, 1}$, Alice Gaudin ${ }^{\ddagger 1}$, Jessica Nakhlé1, Kadi-Liis Veiman ${ }^{1}$, Joël Richard ${ }^{2}$ \\ \& Christophe Chassaing ${ }^{2}$ \\ ${ }^{1}$ Ipsen Innovation, 5 avenue du Canada, Z.I. Courtabœuf, Les Ulis 91190, France \\ ${ }^{2}$ Ipsen Pharmsciences, 20 rue Ethé Virton, Dreux 28109, France \\ *Author for correspondence: Tel.: +33 1609294 44; mathilde.lorscheider@ipsen.com \\ ${ }^{\ddagger}$ Authors contributed equally
}

Global cancer prevalence has continuously increased in the last decades despite substantial progress achieved for patient care. Cancer is no longer recognized as a singular disease but as a plurality of different ones, leading to the important choice of the drug administration route and promoting the development of novel drug-delivery systems (DDS). Due to their structural diversity, therapeutic cancer drugs present specific challenges in physicochemical properties that can adversely affect their efficacy and toxicity profile. These challenges are addressed by innovative DDS to improve bioavailability, pharmacokinetics and biodistribution profiles. Here, we define the drug delivery challenges related to oral, intravenous, subcutaneous or alternative routes of administration, and review innovative DDS, marketed or in development, that answer those challenges.

\section{Graphical abstract:}

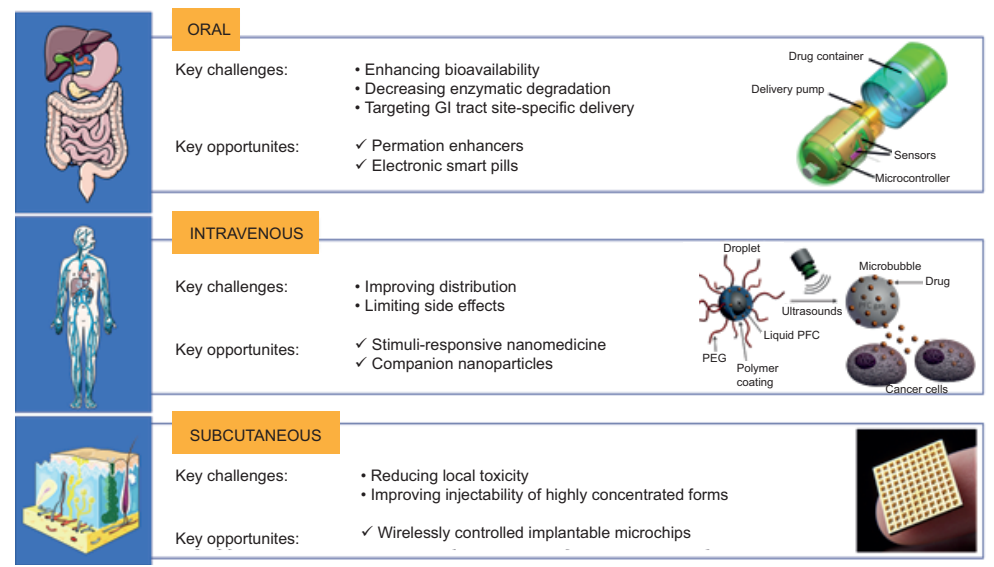

First draft submitted: 25 June 2020; Accepted for publication: 27 November 2020; Published online: 14 December 2020

Keywords: administration routes $\bullet$ cancer drug • drug-delivery systems • innovation • intravenous $\bullet$ nanomedicine - oncology • oral • subcutaneous

Cancer remains the second leading cause of death after cardiovascular diseases, with 9.6 million victims worldwide in 2018, and cancer deaths are projected to increase by $60 \%$ in the next two decades [1]. The pharmaceutical industry has been increasingly focusing its efforts on the research, development and marketing of new oncology drug products to fulfill unmet medical need, leading to the approval of 90 new cancer drugs by the US FDA since 2012 [2]. Among those new drugs, more than 30\% are dedicated to the treatment of blood and lymph node 
(A)

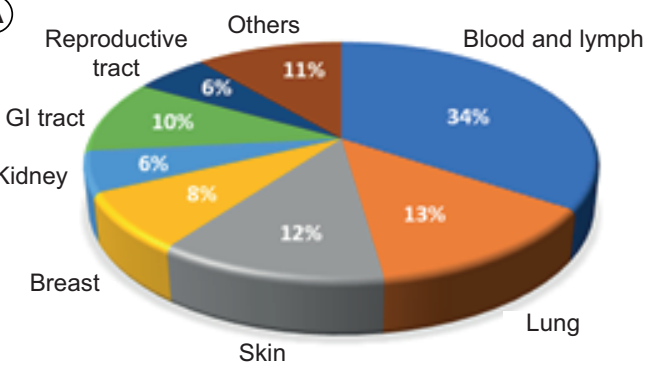

(B)

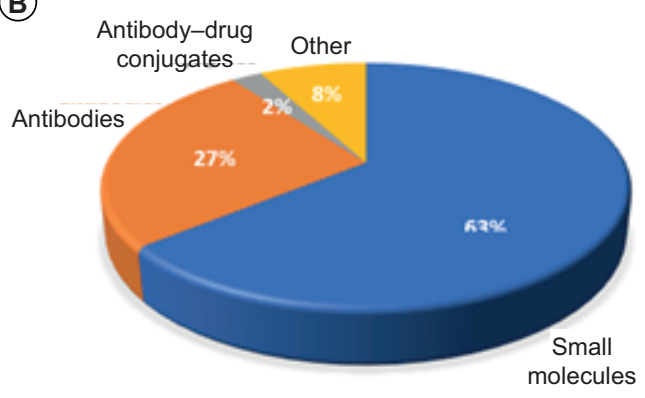

(C)

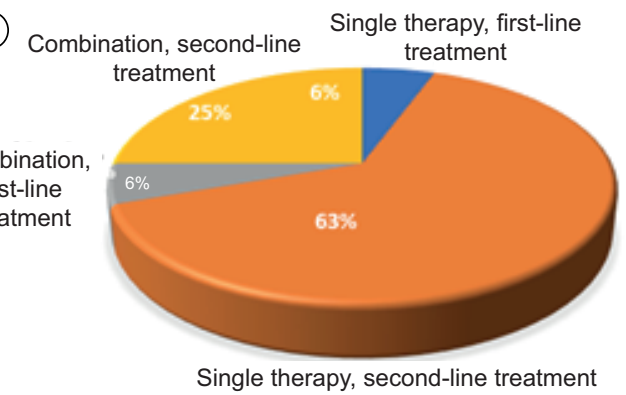

(D)

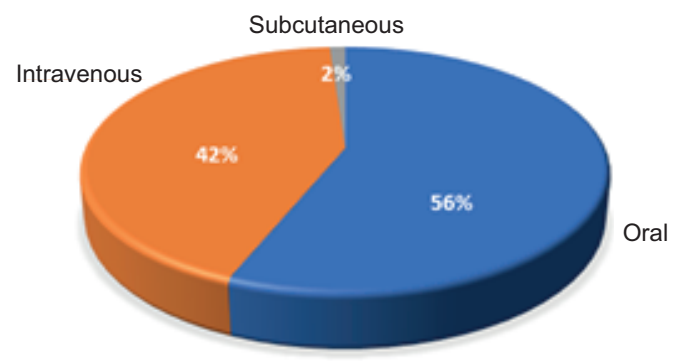

Figure 1. Cancer treatment landscape over the past 5 years. US FDA-approved drugs in oncology since 2012 [2] were classified according to their indication (A), molecule type (B), treatment scheme (C) and administration route (D).

cancers (leukemia, myeloma and lymphoma), and $12-13 \%$ are addressing the treatment of lung and skin cancers (Figure 1A). Other indications represent less than $10 \%$ of the newly approved drugs.

Active pharmaceutical ingredients (API) used for cancer treatments are extremely diverse in terms of chemical structure and pharmacological class, including small molecules, monoclonal antibodies (mAbs) and peptides [3]. Small molecules remain today the major therapeutic class for cancer treatments, representing nearly $63 \%$ of newly approved drugs, although antibodies $(\mathrm{Ab})$ are also a fast-growing market with 23 new Ab-based treatments approved since 2012 (Figure 1B). Within those two therapeutic classes, tyrosine kinase inhibitors, such as Cabometyx ${ }^{\circledR}$, and programmed death receptor-1 (PD-1)-blocking Ab are the two most commonly developed treatment approaches, representing 33 out of 46 small molecules and nine out of 22 antibody-based treatments, respectively. Other therapeutic classes including proteins, systemic radiotherapies or cell-based therapies remain more rarely developed, with the approval of Zaltrap ${ }^{\circledR}$, Xofigo ${ }^{\circledR}$ and $\mathrm{Kymriah}^{\circledR}$, respectively [4]. Most of the current cancer therapeutics commonly used today are in combination regimens. Indeed, around $30 \%$ of the newly approved drugs are administered in combination with one or more other therapeutic agents to increase treatment efficacy (Figure 1C). However, and in order to be successful, these therapeutic entities always require: the selection of the best administration routes for monotherapy and/or for combination and the optimization of the formulation and the drug-delivery systems (DDS) to allow optimal access to the targeted cancer cells.

Different routes of administration are frequently used in cancer therapies, particularly oral administration and intravenous (iv.) administration (Figure 1D), whereas subcutaneous (sc.) administration has very limited application to date. Most of the time, the therapeutic modality used dictates the choice of the administration route. Hence, oral delivery is the gold standard for small molecules, while iv. administration remains the preferred route for more challenging molecules such as biologics, or innovative nano-DDS [5,6]. sc. administration is attractive and generates constant preclinical research but remains less represented in cancer drug late-stage development and approval. Therefore, innovative solutions in formulations and DDS would be required to provide more flexibility and more options in the route of administration, allowing optimal drug delivery to the cancer cells or tissues. For instance, recently, small molecules were formulated in innovative nano-DDS such as liposomes (Vyxeos ${ }^{\circledR}$, Onivyde ${ }^{\circledR}$ and Marqibo ${ }^{\circledR}$ ) or albumin-based nanoparticles (Abraxane ${ }^{\circledR}$ ) to improve access of the drug to the cancer cells and hence increase efficacy of the treatment. The sc. administration was optimized for several Abs such as Xgeva ${ }^{\circledR}$, Herceptin ${ }^{\circledR}$ sc. or Humira ${ }^{\circledR}[7]$ as well as for small molecules $\left(\right.$ Velcade $^{\circledR}$, Synribo ${ }^{\circledR}$ ). Therefore, it appears that 
DDS bring a lot of advantages and opportunities to improve cancer treatment efficacy and access to tumor sites regardless of the molecular class of the API.

Cancer treatments are also facing other challenges. For instance, some APIs have shown poor solubility with limited bioavailability. They may also have a poor stability in the GI tract and poor permeability through the intestinal epithelium making them not suitable for oral dosing. This is the case of large biological molecules, like mAbs, for example, ocrelizumab (Ocrevus ${ }^{\circledR}$ ) or atezolizumab (Tecentriq ${ }^{\circledR}$ ), which are used as immunotherapeutic agents and administrated intravenously to stimulate the cancer immune system. APIs may also present unfavorable physicochemical or pharmacokinetic properties. Small molecules such as paclitaxel (Taxol ${ }^{\circledR}$ ) or doxorubicin (Adriamycin ${ }^{\circledR}$ ) are typically poorly soluble in biological media, requiring chemical modification or formulation development to be administered at their therapeutic dose. These active molecules may also exhibit adverse effects in normal tissues, which would limit their efficacy, due a low therapeutic index. Moreover, many tumor types, such as pancreatic cancer, may be characterized by a very dense stromal compartment or by a poor or unfunctional vascularization making them inaccessible to many kinds of treatments. Considering all these hurdles, there is an increasing demand for effective and, ideally, noninvasive strategies in DDS to ensure the delivery of the right drug, at the right location, over the right period of time while minimizing side effects on healthy cells.

In this review, we present and discuss the challenges and opportunities of the main administration routes and how, when associated with the appropriate DDS, they can address some of the current challenges in cancer drug delivery. Special emphasis will be placed on innovative delivery solutions that could significantly contribute to changing the cancer treatment landscape.

\section{Oral delivery route}

\section{Challenges \& opportunities}

The oral route is usually thought to be the preferred method of administration for patients, as it is the least invasive delivery route, and it complies to cost-containment policies in place in most developed countries, encouraging the move of cancer treatment away from hospital-based settings. Orally administered drugs may be designed to have a systemic effect or a local effect when their targets are in the GI tract. After oral administration, drugs designed to have a systemic effect are expected to enter the bloodstream by crossing the stomach-lining epithelium or more often the intestinal epithelium, and then be subjected to first-pass metabolism by the liver, depending on enzymatic stability [8]. To date, the oral route has been mainly used to deliver small molecules with a molecular weight of less than $500 \mathrm{~g} / \mathrm{mol}$, although new drug-delivery approaches are also considered to expand this delivery route to larger molecules, like peptides and proteins. Three main parameters are driving the bioavailability of small molecules after oral administration: its solubility and stability in intestinal medium and its permeability through the intestinal membrane. The Biopharmaceutical Classification System identifies four classes of drugs, depending on their solubility and permeability. It has been estimated that oral delivery of $90 \%$ of molecules was limited by a poor solubility in the intestinal fluid. Poor oral drug bioavailability is related to four major challenges summarized in Table 1: low drug solubility; poor permeability poor stability, limited absorption and enzymatic degradation of drugs (like e.g., large molecules) in the GI tract; the need for targeted delivery of the drug to specific GI tract sections where cancer is located. The main strategies developed to address those challenges include the development of formulations able to enhance drug solubility and permeability, the use of innovative nanocarriers to improve crossing of the intestinal epithelium, and the development of site-specific delivery systems.

One of the mostly used approaches to address low drug solubility is the drug nanonization process. Drug particle size reduction has been successfully applied to different molecules for many years, allowing the increase of drug substance surface area, consequently enhancing dissolution rate or apparent solubility. This higher apparent solubility improves bioavailability values by a factor of five- to ten-fold [9]. Drug nanocrystals usually exhibit an average size below $1 \mu \mathrm{m}$ and are composed of $100 \%$ drug. Due to their potential physical instability (polymorphism, Ostwald ripening, aggregation...), these nanocrystals may be formulated with stabilizers as solid dosage forms (capsules, tablets) or liquid suspensions. Nanonized drugs are dispersed and stabilized with surfactants or polymeric steric stabilizers forming a drug nanosuspension, avoiding the use of organic solvents or extreme $\mathrm{pH}$ ranges for solubilization and allowing high drug dose in low administration volumes. In addition to an enhanced apparent solubility, increased surface area due to decreased particle size of nanocrystals and/or the presence of surfactants are suspected to promote mucoadhesion, which can increase GI residence time and contribute to increased bioavailability [10]. Many nanocrystal products reached the market since the first drug (Emend ${ }^{\circledR}$ ), for the treatment of nausea and vomiting associated with chemotherapy, was introduced in 2000 [11]. More recently, several molecules 


\begin{tabular}{|c|c|c|c|}
\hline Challenge & Example of drug-delivery solution & Example of technology/product available & Ref. \\
\hline \multirow[t]{2}{*}{ Low solubility/dissolution rate } & Nanonization & NanoCrysta| ${ }^{\circledR}$ & [10] \\
\hline & Lipid-based formulation & SMEDDS & [13] \\
\hline \multirow[t]{3}{*}{ Poor permeability across epithelium } & Permeation enhancers & $\begin{array}{l}\text { Peptelligence }{ }^{\circledR}, \text { Mycapssa }^{\circledR}, \text { Eligen }^{\circledR} \text {, } \\
\text { Rybelsus }^{\circledR}\end{array}$ & [18] \\
\hline & Mucus-penetrating formulations & Covalent coupling to CPP & [27] \\
\hline & Intraepithelial microneedles & RaniPill'M, SOMA & {$[36,38]$} \\
\hline Low drug stability, enzymatic degradation & Encapsulation in nanocarriers & Polyarginine nanocapsules & [33] \\
\hline \multirow[t]{4}{*}{ Gl tract site-specific targeted delivery } & Gastroretentive formulation & $\begin{array}{l}\text { Star-shaped ultralong-lasting capsule, } \\
\text { alginate/polyacrylamide hydrogel }\end{array}$ & {$[40,41]$} \\
\hline & Enteric formulations & Eudragit ${ }^{\circledR}$ & [43] \\
\hline & $\begin{array}{l}\text { Colon-delivery: dual-responsive hydrogels (pH } \\
\text { and microbiota responsive) }\end{array}$ & Guar gum/PVA hydrogel & [47] \\
\hline & Electronic smart pills & Intellicaps ${ }^{\circledR}$ & [52] \\
\hline
\end{tabular}

were approved using the delivery technologies NanoCrystals ${ }^{\circledR}$ from Alkermes, DissoCube ${ }^{T M}$ from SkyePharma and Solumatrix ${ }^{T M}$ from iCeutica. Finally, formulation of poorly soluble drugs such as homogeneous nanosuspensions was shown to minimize inter- and intra-patient variability resulting from fed/fasted state [12], thus bringing a great advantage for chronic treatment of patients with cancer.

Another delivery approach to address poor drug aqueous solubility is lipid-based formulations [13]. Simple oily solutions of complex mixtures of oils and cosolvents, surfactants and cosurfactants can be obtained depending on the drug properties and therapeutic expectations [14]. Microemulsions and self-microemulsifying DDS have been widely explored to maximize the drug solubility in the GI tract. Due to their physical stability as isotropic mixtures systems, the formulations can be filled into hard or soft capsules for oral delivery. These systems spontaneously form lipid droplets in contact with intestinal fluids, achieving a faster and more uniform drug solubilization, permeation and absorption through GI tract. Mostly developed for small hydrophobic molecules, self-microemulsifying DDS could also be formulated with therapeutic peptides and proteins. However, because of their mainly hydrophilic nature, drug loading is usually limited in lipidic formulations, unless hydrophobic ion pairing strategies and additional formulation optimization routes are used [15]. Commercial successes were achieved with the formulation of the immunosuppressant peptide cyclosporine $A\left(\mathrm{Neoral}^{\circledR}\right)$, or antiretroviral drugs ritonavir $\left(\right.$ Norvir $\left.^{\circledR}\right)$, saquinavir $\left(\right.$ Fortovase $^{\circledR}$ ) and tipranavir (Aptivus ${ }^{\circledR}$ ), showing the benefits of that type of DDS for cancer therapeutics applications.

These lipidic systems combined with functional molecules known as permeation enhancers have the major advantage of addressing both solubility and permeability issues [16]. The key issue associated with this strategy is to ensure the simultaneous codelivery of the drug substance and the permeation enhancer at the absorption site to achieve significant increase in bioavailability without inducing toxicity. Typically, drugs requiring permeation enhancement strategies are peptides, peptide analogs or other polar high-molecular-weight molecules. The most promising technologies were developed up to late clinical stage, such as Peptelligence ${ }^{\circledR}$ from Enteris BioPharma and Transient Permeation Enhancement (TPE ${ }^{\circledR}$ ) from Chiasma. Both technologies are based on enteric-coated solid dosages forms, combining the drug substance with a permeation enhancer, acyl carnitine for Peptelligence ${ }^{\circledR}[17]$ and sodium caprylate for TPE ${ }^{\circledR}$. The simultaneous release of the permeation enhancer and the peptide drug increases its solubility and absorption by improving passive paracellular transport through the tight junctions (TJ). Chiasma recently announced acceptance by FDA of its new drug application resubmission for oral octreotide capsules (Mycapssa ${ }^{\circledR}$ ) for the treatment of neuroendocrine tumors (NET) and acromegaly [18], a rare, debilitating disease caused by a benign tumor of the pituitary gland. Moreover, Rybelsus ${ }^{\circledR}$ developed by NovoNordisk based on the Eligen ${ }^{\circledR}$ technology was FDA-approved in September 2019. The oral delivery of the GLP-1 analog is achieved by the creation of a noncovalent complex between the drug and the salcaprozate sodium protecting the peptide from digestive enzymes and improving its permeability through the intestinal epithelium via a transcellular pathway. This marketed technology could be adapted to oncology peptides, proteins or other large molecules $[19,20]$. 
Upcoming challenges for oral administration appear to be the delivery of fragile molecules, such as peptides or proteins, due to their extremely low stability in the GI tract and resulting in poor bioavailability [21]. Several strategies to enhance stability and permeability of these molecules are currently explored at earlier stage of development [22]. Chemical modification of drugs to form prodrugs can be achieved by covalently linking nonpharmacologically active molecules to the drug to increase their permeability and reduce their tendency to metabolism [23]. Among others, the two strategies evaluated in early development phases were covalent coupling with poly(ethylene glycol) (PEG), known as PEGylation [24], or with cell-penetrating peptides (CPPs), such as the transactivator of transcription of HIV [25]. The most critical aspects in this approach are the identification of functional reactive groups for covalent modification of the drug without modifying its pharmacological activity, and the selection of a linker strategy. This strategy ensures the conjugate stability in the GI tract, while allowing drug release in a timely manner and therapeutically active state at the site of action. Those advantages are key in cancer therapy for the delivery of peptides and proteins. Other examples of CPPs can be cited as transepithelial carriers for the delivery of proteins or peptides such as polyarginine and penetratin, which were mostly used to deliver insulin [26-28]. Chemical modification of drug substances was also developed in the squalenoylation platform [29], in which small hydrophilic drugs are coupled to the lipidic squalene to form an amphiphilic prodrug able to self-assemble and form nanoparticles. This strategy has been applied to the oral delivery of cisplatin [30].

The use of nanocarriers has also been extensively explored to enhance drug bioavailability after oral administration due to the nanoscale size of the carrier and the increased epithelial permeability related to a specific interaction mechanism or cell-penetrating properties and many examples in preclinical development stage could be cited here. One of them are the polyarginine (PArg) nanocapsules were specifically designed to deliver small anticancer drugs into cells and were recently used in early development for the oral delivery of an antitumoral peptide, elisidepsin. These nanocapsules are composed of an oily core of Mygliol ${ }^{\circledR}$ and lecithin, and a PArg polymeric shell whose guanidine functional groups, well known as CPPs, promote cellular internalization. The PArg nanocapsules demonstrated prolonged retention in the GI tract following oral administration in mice.

Due to their interaction with the GI mucosa, they were likely able to develop interactions with TJ proteins that would favor paracellular transport of hydrophilic drugs. Despite significant enhancement in bioavailability compared with free drugs, the biggest challenge for those systems remains the permeation through the intestinal membrane to reach the systemic circulation. Without proper surface modification such as the addition of CPP moieties, due to their nanometer size range, nanocarriers can encounter difficulties to penetrate the intestinal mucus, especially in the thickest parts of the GI tract walls [31,32]. Hence, optimization regarding size and surface properties of nanocarriers are necessary to ensure they efficiently infiltrate the mucus layer before turnover occurs [33].

Oral nanocarriers also reached clinical development stages such as eRAPA, the encapsulated rapamycine currently investigated in Phase II clinical trial for the treatment of nonmuscle invasive bladder cancer [34]. eRAPA is a submicronic particle composed of poly(methyl metacrylate), a $\mathrm{pH}$-sensitive polymer. Rapamycine given orally as a free drug encounters a short half-life combined with a strong first-pass hepatic resulting in very low bioavailability. The innovative encapsulated rapamycine, eRAPA, showed an increased bioavailability and promising safety of the drug in clinical Phase $\mathrm{Ib}[35]$.

When reaching the intestinal lumen, some anticancer drugs face poor mucus penetration. To address this challenge, several innovative delivery systems are currently under investigation preclinically and clinically. These systems are based on the use of dissolvable drug-loaded microneedles embarked in smart devices placed in gastroresistant capsules. When reaching the intestinal section of the GI tract, the microneedles are inserted into the intestinal epithelium. The drug loaded inside the microneedles is then released upon dissolution and reaches the systemic circulation. This is the case with the RaniPill ${ }^{T M}$ system, developed by Rani Therapeutics [36]. This system is currently under preclinical evaluation for the delivery of several peptides and proteins and demonstrated bioequivalence to SC injection with a GLP-1 analog, PTH and a TNF- $\alpha$ inhibitor. The first-in-human safety study was successfully completed in March 2019 focusing on the tolerability of the device with no drug loaded [37]. The major limitation of this technology is the low drug loading of 3-5 mg of API per pill. This might be an issue when evaluating small molecules for cancer treatment, which usually require higher doses. Another example is the selforienting millimeter-scale applicator (SOMA) device developed by MIT in collaboration with NovoNordisk, at a preclinical stage for the oral administration of insulin. This promising device demonstrated similar pharmacokinetics profiles of the insulin compared with standard SC administration [38].

Apart from systemic delivery, oral route of administration can be used as a local delivery route of the drug substance to a GI site-specific tumor such as gastric, intestinal or colorectal cancer. To address this need, several 
drug-delivery strategies are currently being evaluated. Stomach is the easiest GI section that can be reached after oral administration of a drug. However, the drug residence time in the stomach is usually short and therefore may limit the therapeutic efficacy of the delivered drug. Most advanced systems include gastroretentive dosage forms, to increase the gastric residence time [39]. One of those systems, developed for a noncancer treatment, is an ultralong-acting capsule that deploys a star-shaped dosage form preventing its transit through the pylorus, and delivers a sustained therapeutic dose of ivermectin against malaria up to 14 days [40]. Another system is based on two types of alginate and polyacrylamide networks that are interpenetrated and cross-linked, forming an hydrogel with significant swelling and mechanical properties allowing to withstand in vivo gastric forces [41]. Interestingly, this system can be dissolved and eliminated on demand, by simply swallowing a biocompatible chelator and a reducing agent. This property would be an absolute necessity in the case of, but not limited to, cancer therapy, to be able to stop the treatment in case of any adverse reaction. The major challenge faced when targeting an intestinal cancer is to resist the harsh milieu of the stomach and deliver the drug only in the more neutral environment of the intestine. Such properties are generally achieved using enteric-coated formulations formed with polyanionic $\mathrm{pH}$-sensitive polymers that dissolve only at neutral $\mathrm{pH}[$ [42]. Cellulose derivatives such as cellulose acetate, phthalate or methacrylate copolymers (e.g., Eudragit ${ }^{\circledR}$, polymers from Evonik) were shown to efficiently protect drugs from the acidic environment of the stomach allowing drug release in the intestinal fluid [43].

Oral delivery may also give the opportunity to reach the intestinal lymphatic system, which can be extremely useful for the treatment of lymphoma and some metastatic cancers [44]. This delivery route that has the advantage of circumventing the first pass effect, would be attractive for drugs sensitive to liver metabolism but the bioavailability of the molecules is relatively low through this route of absorption. Since the intestinal lymphatic system plays a major role in the absorption of dietary lipids via phagocytosis of chylomicrons, most strategies to target this system are using lipid-based formulations and chemical modifications of drugs to form lipid prodrugs [45].

Finally, drug delivery targeting the colon part of the GI tract may be used to improve delivery to colorectal tissues, especially in the case of colorectal cancers [46]. This strategy can also improve systemic absorption of macromolecules, due to lower proteolytic activity, decreased expression of efflux transporters and increased residence time. Delivery to the colon faces two challenges: the drug needs to reach the colon without being released neither in the stomach nor in the intestine, and to cross the thick mucus layer present in this part of the GI tract [47]. Guar gum, a natural polysaccharide is solely and specifically degraded by the colon anaerobic microbiota into monosaccharides, has been used to deliver the anticancer drug, 5-fluorouracil (5-FU) [48]. It could form a pH-responsive hydrogel when combined with polymers such as poly(vinyl) alcohol [49]. However, these strategies need to be carefully adjusted to the disease state, which can easily modify both the $\mathrm{pH}$ and the microbiota of the colon.

The next generation of oral drugs will likely take advantage of innovative technologies such as $3 \mathrm{D}$ printing [50]. Beyond the possibilities of rapid prototyping, 3D printing offers unprecedented flexibility in the design and manufacturing of drugs, which is particularly well suited for personalized medicines. Hence, $3 \mathrm{D}$ printing is gaining growing interest in the pharmaceutical field, especially since the FDA approval in 2015 of Spritam ${ }^{\circledR}$ (levetiracetam) 3D-printed tablets for the treatment of epileptic seizures [51]. The Spritam tablet is built by powder liquid layerby-layer deposition until the desired dosage is achieved. It allows for a high dose of medicine to be loaded into a relatively compact pill. The pill is porous, enabling it to disintegrate quickly with a sip of liquid to make it easier for patients who have trouble swallowing and to comply with their therapeutic regimen. Looking at the near future, innovative drug delivery digital devices are also currently explored for personalized therapy. This includes the electronic smart pill Intellicap ${ }^{\circledR}$ from Medimetrics Personalized Drug Delivery, which can measure individual GI residence times, temperature and local $\mathrm{pH}$, and communicate the results in real time via a wireless transceiver [52]. This should allow determining the location of the capsule, and tightly and remotely controlling drug delivery to a targeted region. This smart drug delivery pill will likely enable a range of novel therapies and transform the conventional oral delivery pill to a key building block of a future personalized and interconnected healthcare environment.

\section{Conclusion on oral delivery of anticancer drugs}

Challenges and examples of drug-delivery solutions for the oral administration of anticancer drugs are summarized in Table 1 . The oral route remains the preferred mode of delivery for most drug substances, owing largely to simplicity and noninvasiveness and improving quality of life of patients with cancer [53]. This route of administration makes perfect sense for the treatment of gastrointestinal and colorectal cancers, since it ensures a direct access to the tumor knowing that the drug absorption in the colon can present some variability. Moreover, the dosage form 
developed must be appropriate for a localized delivery requiring complex and expensive technologies such as smart pills. This route can also provide a specific entry into the systemic lymphatic system through the intestinal lymphatic network, relevant in the context of lymphoma and in some cases of metastatic cancers, using lipid-based formulations. It is also commonly used in multiple other indications such non-small-cell lung cancer, renal cell carcinoma or hepatocellular carcinoma. However, systemic biodistribution via oral administration still needs to overcome numerous challenges to improve bioavailability and fully compete with IV administration, especially for the delivery of large molecules such as peptides and biologics. When developing a novel oral formulation, it is also critical to consider the usually high dose of macromolecules or small molecules to be administered and adapt the technology to this challenge [54]. For cytotoxic agents with a narrow therapeutic window, oral administration might be limited by intra- and interpatient absorption variability, and the necessity to closely monitor the treatment regimen and tolerability. Conversely, more recent cancer drugs such as noncytotoxic-targeted agents or hormonal therapy, as described earlier, involving therapeutic molecules with wider therapeutic windows, may largely benefit from novel oral formulations such as the nanotechnologies [55,56].

\section{iv. delivery route}

\section{Challenges \& opportunities}

iv. administration refers to the direct injection - infusion or bolus - of drugs into the bloodstream, allowing direct access to any organ vasculature in the body. iv. injections ensure higher bioavailability and lower interand intra-patient variability compared with the oral route. However, this delivery route for drug cancer therapies still faces four main challenges to significantly improve patient quality of life: long infusion times are usually required due to poor pharmacokinetics and biodistribution properties of the drug substance, in other words, rapid plasmatic clearance, strong metabolism, fast excretion or short residence time at the tumor site. Conventional chemotherapeutic agents are usually nonspecific and distribute both in normal and tumor tissue, leading to severe side effects and off-target toxicity therefore requiring targeted therapies to increase therapeutic efficacy. Moreover, targeted therapies could help reach some isolated organs due to specific biological barriers, such as the blood-brain barrier (BBB) for brain targeting. Some other cancer therapies, such as immunotherapies or targeted therapies, may also have a broader expression of the target, not only limited to tumor sites but also present in multiple normal organs, leading to undesirable side effects (toxicity on targets present in healthy organs not containing any tumor). Finally, treatment strategies that need to be fine-tuned to the patient and to the tumor sites only, usually involve the use of combination therapies and imaging modalities.

Hence, the development of highly versatile and modular formulations is required to address the challenges described above. Although other technologies such as the synthesis of bioconjugates have been implemented, the main innovative strategies that will be described in the following sections are based on the use of nanotechnologies applied to the therapeutic field, usually referred to as nanomedicine [57]. Due to its versatility, nanotechnology could be one common answer to the four challenges stated above.

Initial interest in nanocarriers has been largely related to their ability to increase drug substance circulation time and modify tissue distribution profile due to their size and surface properties [57], therefore addressing the key challenge of unfavorable pharmacokinetics, biodistribution and toxicity profiles of iv. cancer drugs.

To achieve this, nanocarriers used as nanosuspensions, such as liposomes, nanoparticles or nanocapsules, were developed. Longer circulation times were obtained, thanks to surface modifications, mostly using PEG, to limit opsonization and the subsequent rapid capture and clearance by the macrophages of the reticuloendothelial system [58,59]. The PEG, an hydrophilic FDA-approved polymeric excipient, is widely used as a surface modifier of macromolecules and particulates to improve pharmacokinetics and pharmacodynamics properties [58]. For example, PEGylated phospholipids were used in the composition of Doxil ${ }^{\mathbb{R}}$, a liposomal formulation of doxorubicin. In patients, the total body exposure, expressed as area under the curve, of the encapsulated doxorubicin was 300 -fold greater than the free drug demonstrating the significant impact of the PEG chains at the liposome surface [60]. However, PEG itself presents the disadvantage of a nonbiodegradable main chain, and anti-PEG immune responses have been observed and correlated to loss of therapeutic activity and adverse reactions in several clinical reports after chronic administrations [61]. In addition to the formation of Ab following multiple administrations of PEGylated therapeutics, the pre-existence of anti-PEG Ab in the general population exposed to PEG through other means can also contribute to the overall immune response [62-64]. These findings bring to light a need for improved awareness regarding anti-PEG Ab [65], especially in the drug delivery field [66]. Interestingly, over the past decades, alternatives to PEG have been investigated at the research stage to increase the circulation half-life of nanocarriers [63], such 
as the use of polyglycerols [67], polysialic acid [68] or polyamino acids [69], although these molecules have not yet reached the market as nanocarrier surface modifiers due to less promising effects on improving drug circulation half-life [70]. As long-circulating stealth nanocarriers are obtained due to their surface modification, the control over tissue distribution is primarily based on their nanometric size (usually $<200 \mathrm{~nm}$ ), allowing passive accumulation within the tumor mass through the enhanced permeability and retention (EPR) effect. It was demonstrated the passive accumulation of nanocarriers is usually related to tumor physiology, especially its leaky vasculature, the poor lymphatic drainage [71] and the increased inflammatory tumor response [72]. Similarly to nanoencapsulation, the nanonization of drugs to form nanosuspensions [10] and the development of albumin-based drug nanoparticles [73] were implemented to enhance loading capacity of insoluble compounds and reduce infusion time and volume. Therefore, nanotechnology takes the advantage of the cancer pathophysiology to passively address the carriers to the tumor site. Although the EPR effect has become the foundation of nanocarriers delivery to solid tumors, this effect presents a high variability among tumor types, tumor development stage [74], tumor microenvironment and stroma characteristics [71]. Within one tumor type, several strategies were proposed to take interpatient variability into account and select patients according to their EPR capability, such as the use of companion imaging particles [75], the development of radiolabeled nanocarriers [76] or the evaluation of circulating EPR-predictive gene, protein or cell biomarkers [77]. Tissue distribution of encapsulated drugs can also be significantly improved if a component of the nanocarrier (ligand) specifically interact with a molecule overexpressed in the tumor, providing active targeting. This strategy led to the FDA approval of Abraxane ${ }^{\circledR}$ for the delivery of paclitaxel in 2005 for the treatment of pancreatic ductal adenocarcinoma, using albumin-based paclitaxel nanoparticles (Abraxane ${ }^{\circledR}$ ). In this context, a glycoprotein, SPARC (secreted protein acidic and rich in cysteine), is highly expressed in the tumor stroma, and it has been suggested that this protein could sequester Abraxane ${ }^{\circledR}$, enhancing the delivery of paclitaxel into the tumor microenvironment [78]. Although the associations between Abraxane ${ }^{\circledR}$ and SPARC remain to be fully demonstrated, several clinical trials are currently ongoing in order to investigate the combination of Abraxane ${ }^{\circledR}$ with chemotherapies (in particular gemcitabine), or immunotherapies, for the treatment of pancreatic ductal adenocarcinoma [79]. Active targeting can also address the tissue distribution challenge for the delivery of cancer therapies to cross cellular membranes and reach intracellular targets [80]. To answer this challenge, nanocarriers were designed to improve specific cellular uptake, usually with targeting ligands that recognize specific receptors on the tumor cell surface [81]. Several companies carried out clinical trials on targeted nanoparticles such as BIND Therapeutics Inc., evaluating in Phase II the targeted polymeric nanoparticle BIND-014 against prostate cancer, non-small-cell lung cancer and cervical cancer [82]. BIND-014 is a Prostate-Specific Membrane Antigen (PSMA)targeted nanoparticle formulation encapsulating docetaxel. PSMA is a protein specifically expressed on prostate cancer cells surface and in the tumor neovasculature but not on healthy tissue vasculature. This specific expression of PSMA was used as a target-ligand couple for the tumor cell internalization of the docetaxel-loaded nanoparticle [83]. Similarly, Synergene Therapeutics is currently evaluating transferrin-targeted immunolipoplexes encapsulating p 53 DNA sequence in Phase II clinical trials for the treatment of pancreatic cancer and glioblastoma [84]. Synergene Therapeutics exploited the increased expression of transferrin receptors at the BBB for an efficient and specific targeting of the brain parenchyma. A major barrier in pharmacology remains the BBB that prevents more than $98 \%$ of endogenous and exogenous molecules circulating in the bloodstream to reach the brain [85]. Noninvasive brain delivery strategies such as the one of Synergene Therapeutics aims to exploit existing pathways to cross the BBB, by either forming prodrugs able to cross or circumvent the barrier [86], or by encapsulating the drug into nanocarriers decorated with a BBB-targeting moiety $[87,88]$. However, regardless of the type of drug encapsulated in the past decades, no more than $1 \%$ of the injected dose has reached the brain parenchyma so far, when using such targeted and multifunctional nanocarriers. Overall, a thorough understanding of the surface charge interactions between the nanocarrier and biological systems, especially circulating proteins and cells, is needed before translating passive or active targeting strategies to the clinic. Increased uptake by tumor cells could also be achieved by exploiting size- and shape-dependent cell internalization mechanisms [89,90]. These aspects are currently highly explored at a preclinical stage on metal nanoparticles (Gold, Silver, Copper) [91-93], as well as on organic and biodegradable polymer nanoparticles [94,95]. As an example, this challenge has been addressed by specifically printing nanoparticles with precisely defined size and shape and evaluating preclinically their fate after iv. administration [96,97]. Liquidia Technology translated the PRINT technology from research to cGMP manufacturing scale with two current clinical programs for noncancer diseases [98]. They demonstrated the versatility of the technology versus the molecule type and the route of administration. Most of the recent examples highlighted above on innovative nanomedicines have been developed for the encapsulation of small molecules or macromolecules (proteins, peptides...). A 
relatively more recent challenge associated to nanodrug delivery is the encapsulation of gene therapy (DNA, RNA sequences) to target intracellular compartments. Two main nanocarrier families were investigated: the viral vectors and the nonviral vectors (liposomes, polymer or lipid nanoparticles) already described above with their associated challenges [99,100]. Viral vectors, mainly retroviruses, adenoviruses and lentiviruses structures, are known for their efficient and fast cellular uptake and their capacity to deliver the cargo to the intracellular target. Their ability to overcome extracellular and/or intracellular barriers due to their specific structure, size, shape and surface properties. However, safety concerns were highlighted in preclinical and clinical studies with the apparition of off-target immunogenicity, inflammatory response and toxicity [101,102].

Many targeted therapies have to face the issue of side effects due to the ubiquitous distribution of the targeted receptor in healthy organs also, even if usually overexpressed at the tumor site. To address this challenge and reduce the drug concentration in healthy tissues while retaining the antitumor activity, the drug release can be precisely controlled by using a biological stimulus specific from the targeted site. The Probody ${ }^{\circledR}$ technology platform (CytomX ${ }^{\circledR}$ Therapeutics) designed therapeutic prodrug-Ab to be specifically converted by enzymatic activity at the tumor location from a nonactive entity to its active form. The modified $\operatorname{IgG}$ antibody was transformed in the Probody ${ }^{\circledR}$ inactive form with the addition of a masking peptide linked to the light chain $\mathrm{N}$-terminal via a proteasecleavable linker. After IV administration, once the Probody ${ }^{\circledR}$ construct is located in the tumor extracellular matrix, the masking peptide is removed through the cleavage of the linker, therefore releasing the peptide [103,104]. Due to the overexpression of the converting protease in the tumor environment, the nonactive $\mathrm{Ab}$ is specifically activated at the tumor site, reducing on-target side effects and increasing the benefit/risk ratio of the drug [105]. Finally, control over drug distribution can also be achieved by precisely controlling drug release using stimuli-responsive nanocarriers [106]. External stimulations such as heat, light, magnetic field or ultrasounds enable tailored drug-release profiles with time and space control. This external stimulus is fully independent from any biological stimulus $(\mathrm{pH}$, redox state or enzyme activity) that could introduce an additional inter- and intra-patient variability. For example, the thermosensitive liposomal formulation of doxorubicin, ThermoDox ${ }^{\circledR}$, is currently explored in a Phase III trial for the treatment of nonresectable hepatocellular carcinoma [107]. ThermoDox ${ }^{\circledR}$, delivered by iv. infusion, is used in combination with heat-producing techniques, typically microwave hyperthermia or high-intensity-focused ultrasound applied on the area to be treated. Reaching a temperature above the lipid mixture glass transition, usually around $40-45^{\circ} \mathrm{C}$, the structure of heat-sensitive bilayer becomes more fluid, creating openings in the liposome. This fluidity enables doxorubicin release specifically into and around the targeted area [108].

Similar to this approach, the use of ultrasound-mediated drug delivery in combination with gas microbubbles of $1-10 \mu \mathrm{m}$ in diameter could be a promising strategy to reach the brain parenchyma. Gas microbubbles mechanically disrupt BBB TJ providing potential for enhanced anticancer treatment, especially in terms of spatial specificity [109]. In this setting, drugs can be formulated either directly inside the microbubbles used to disrupt the junctions, or in adjunction of free molecules, or encapsulated in another type of nanocarrier. Interestingly, this strategy can be coupled to MRI, to monitor BBB permeation [110] and provide a noninvasive theranostic tool [111]. Finally, as ultrasounds can also be used to generate heat and disrupt thermosensitive liposomes such as previously described for ThermoDox ${ }^{\circledR}$, using this technique could provide a way to both locally deliver the liposomes and release the drug in a timely manner. This DDS presents advantages in noninvasive imaging and the delivery of drug in neurological disorders and brain cancer but the safety has to be monitored closely due to potential thermal and mechanical injuries in the $\mathrm{BBB}$, as well as risks of inflammation, hemorrhages and functional effects $[112,113]$.

As stated before, cancer monotherapy had so far limited clinical benefits due to drug-resistance or dose-limiting toxicity. As an alternative, combination therapies of chemotherapy drugs were applied for years in clinical practice, combining different therapeutic approaches such as cytotoxic small molecules, immunotherapy, targeted therapy or gene therapy. The goal of combination therapy is to obtain a synergistic effect, to achieve maximal therapeutic efficacy and minimal toxicity. In this context, the main challenge is to ensure the delivery of each drug component with the appropriate pharmacokinetics and distribution, and the optimal drug-to-drug ratio [114]. Combined therapies can be provided as the simple administration of two or more standard chemotherapies, or the coadministration of one standard molecule with an encapsulated one, or the coencapsulation of the molecules in one unique nanosystem. Coencapsulation provides the highest control over site and time delivery of both components, thus increasing intracellular drug concentrations within one cell, and coordinating drug synergy. Due to their structural versatility, nanocarriers offer the possibility to coencapsulate multiple drugs within a single nanomedicine. One combination treatment, Vyxeos ${ }^{\circledR}$, was recently approved as a liposomal coformulation of daunorubicin and cytarabine for the treatment of newly diagnosed therapy-related acute myeloid leukemia [115]. The two drugs are delivered 


\begin{tabular}{|c|c|c|c|}
\hline Challenge & Example of drug-delivery solution & Example of technology/product available & Ref. \\
\hline Short half-life, fast metabolism and rapid elimination & PEGylation & $\operatorname{Doxil}^{\circledR}$ & [60] \\
\hline \multirow[t]{4}{*}{ Nonspecific distribution to healthy tissues } & Albumin-based nanoparticles & Abraxane $^{\circledR}$ & [79] \\
\hline & Targeted nanoparticles & BIND-014/Accurins ${ }^{\circledR}$ technology & [82] \\
\hline & Nonspherical nanoparticles & PRINT $^{\circledR}$ technology & {$[96,97]$} \\
\hline & Thermosensitive delivery & ThermoDox ${ }^{\circledR}$ & [107] \\
\hline Limited therapeutic efficacy of monotherapy & Coencapsulation & $\operatorname{Vyxeos}^{\circledR}$ & [115] \\
\hline \multirow[t]{3}{*}{$\begin{array}{l}\text { Necessity to stratify patient and follow-up treatment } \\
\text { responsiveness (theragnostic) }\end{array}$} & $\begin{array}{l}\text { External stimuli to open the biological } \\
\text { barrier }\end{array}$ & Ultrasounds and microbubbles & [109] \\
\hline & Systemic radiotherapy & $\begin{array}{l}\text { Somatostatin analog linked to a chelator } \\
\text { cage filled with }{ }^{68} \mathrm{Ga} \text { or }{ }^{177} \mathrm{Lu}\end{array}$ & [116] \\
\hline & 'All-in-one' nanocarrier & Nanoporphyrin & [119] \\
\hline
\end{tabular}

simultaneously in a unique formulation to the patient, decreasing volumes of administration and improving patient compliance.

Combination therapies may also integrate either a diagnostic tool or a therapeutic approach into a single formulation to form a theranostic agent. Such theranostic strategy is currently explored with the development of systemic radiation therapies (SRT). One example of SRT development is the coupling of a somatostatin analog with a chelator cage filled with a radionuclide-emitting beta radiation, in the context of metastasized NET. Depending on the chelator cage, ${ }^{68} \mathrm{Ga}$ or ${ }^{177} \mathrm{Lu}$ is used for diagnostic or treatment, respectively [116]. Lutathera ${ }^{\circledR}$ (lutetium 177Lu dotatate) was FDA approved as the first systemic radiation therapy in 2018. It is indicated for the treatment of somatostatin receptor-positive gastroenteropancreatic-NET, a rare group of cancers with limited treatment options $[117,118]$. Given the possibility to codeliver different molecules, nanomedicines were also explored to develop theranostic agents. An example of such system is a highly versatile 'all-in-one' porphyrin-based organic nanoconstruct (nanoporphyrin) made of a single organic building block (a porphyrin/cholic acid hybrid polymer), and able to integrate a variety of imaging (NIR-fluorescence, MRI, PET...) and therapeutic functions in a coreshell structure [119]. However, clinical development of such complex nanocarriers for the theranostic approach poses new challenges stemming from the escalating complexity in chemistry, manufacturing and controls [120]. Moreover, those systems will likely require the development of new assays and regulatory standards to ensure controllable and reproducible synthesis as well as consistent in vivo performances [121].

\section{Conclusion on iv. delivery of anticancer drugs}

Challenges and examples of drug-delivery solutions for the iv. administration of anticancer drugs are summarized in Table 2. iv. administration remains the main delivery route for anticancer treatments since it ensures controlled bioavailability and low interpatient variability. This delivery route requires the intervention of a nurse or a physician to administer the drug. The patients are therefore closely followed-up by healthcare professionals and side effects or drug-drug interactions are accurately monitored. However, patient quality of life and compliance associated with this delivery route suffer from the low therapeutic window of most anticancer drugs, due to poor stability in circulation and low specificity to tumor tissues, which induces toxicities and on-target or off-target side effects. Nanomedicine has offered innovative solutions, some of them already implemented in the clinic, especially to increase circulation time and promote tumor accumulation (Table 2). Due to their versatility, nanocarriers also offer great promises regarding the delivery of fragile biomolecules, as well as the implementation of combination treatments, theranostic strategies and personalized approached. Those very innovative approaches lead to additional development costs, which are reflected in the final price of the marketed drug. Depending on the healthcare policy of each country, this can be an important issue in terms of cost reimbursement or even access to care.

\section{sc. delivery route}

\section{Challenges \& opportunities}

sc. injection is usually performed using a wide variety of needle gauge (18-30G) and can be completed by patients themselves, such as in the case of the autoinjection of diabetes chronic treatment. Upon sc. injection, as described in the literature, low-molecular-weight molecules $(<16 \mathrm{kDa})$ can diffuse and reach the systemic circulation through 
capillaries, whereas absorption through the lymphatic network is the predominant pathway in the case of larger molecules [122-124]. Therefore, sc. bioavailability can be variable, compared with the iv. route depending on the physicochemical properties of the drug substance. The usual injection volume in the sc. compartment is $1.5 \mathrm{ml}$ and volumes higher than $2.5 \mathrm{ml}$ are considered to be associated with pain and adverse events at the injection site [125,126]. Therefore, it requires highly concentrated formulations to deliver the right therapeutic doses to the patient [127]. Highly concentrated solutions or suspensions usually present a high viscosity that results in poor injectability and consists in one of the main challenges of this delivery route. As an alternative to iv. injection, sc. injection is usually dedicated to chronic treatments. To limit the number of administrations, the second challenge is to develop sustained-release formulations to prolong drug release over longer time periods without inducing local toxicity due to the high activity of anticancer drugs. Ideally, one would also like to control the rate of drug release to allow drug combinations or specific treatment schemes in a single formulation. The main strategies to address those challenges rely on the use of polymer mixtures with different physicochemical properties to create injectable suspensions of microparticles/microcapsules, soluble polymer-prodrugs, polymeric implants or polymeric liquid gels, liquid in situ forming implant formulations, as well as the development of implantable microchips able to deliver drugs on demand through closed loop systems or wireless control. These formulations were successfully developed for the past 15 years, mostly to deliver small molecules and peptides.

Different technologies were explored to address the challenge of injectability of highly concentrated peptide formulations. For example, the FluidCrystal ${ }^{\circledR}$ technology (Camurus) is currently being evaluated for the delivery of the peptide octreotide in a Phase III clinical trial [128]. The FluidCrystal ${ }^{\circledR}$ technology relies on the solubilization of the peptide in a nonaqueous solution containing lipids, glycerides and organic solvents, which is administered as an organic depot using small needles of 23-27G. In contact with the aqueous environment of the subcutis, the depot self-organizes in situ into a liquid crystal matrix, able to slowly release the drug upon lipase degradation and diffusion. Hence, this technology combines both advantages of being a ready-to-use formulation, with a low viscosity at the time of administration. An in situ forming implant, Eligard ${ }^{\circledR}$, was also developed for the delivery of leuprolide as a palliative treatment for advanced prostate cancer [129]. The leuprolide is mixed with biocompatible organic solvent (NMP) and biodegradable polymers (poly(lactic-co-glycolic) acid, PLGA) and administered as a liquid that solidifies in the body due to diffusion of the solvent in the SC environment followed by polymer precipitation. The slow release of the drug over time is dependent on the polymer degradation rate by usual biologic processes. Since solidification only occurs after administration, this system significantly improves the injectability of the treatment [130]. Similarly, the sc. delivery of $\mathrm{mAbs}$ also requires high concentrated formulations to reach therapeutic efficacy. High concentrations of mAb solutions were achieved, using crystalline suspensions [131] or gel beads [132]. The derivatization of Abs or proteins as PEG conjugates was also described for their sc. administration to increase their hydrodynamic diameter, reduce the kidney excretion and enhance their lymphatic uptake [133], such as in the case of Somavert ${ }^{\circledR}$, a PEG-HGH antagonist marketed by Pfizer for the treatment of acromegaly [134]. The SC administration can also take advantage of intrinsic properties of the drug substance. Sustained-release highly concentrated formulations of peptides are achieved by exploiting peptide self-assembly properties [5], like in the case of Somatuline ${ }^{\circledR}$ Autogel ${ }^{\circledR} /$ Depot. Despite its inherent viscosity, this simple self-assembled semisolid formulation of lanreotide acetate forming nanotubes is a unique highly innovative ready-to-use marketed product for the treatment of acromegaly and gastroenteropancreatic-NET [135].

In systemic treatments, the therapeutic properties of the drug are mostly controlled by the rate and extent of drug absorption, rather than the administered dose [136]. Hence, numerous sustained-release systems were developed over the years, to tightly regulate release profiles and maintain drug concentration within an optimal therapeutic range for prolonged duration of treatment. The sc. space is a unique local environment where such systems can be implanted to form a depot able to steadily release the drug over time. Decapeptyl ${ }^{\circledR} S R$, a prolonged release formulation of triptorelin for the treatment of prostate cancer based on PLGA microparticles, was the first product to reach the market by exploiting such specific properties of the SC environment [137]. Formulating PLGA microparticles of the hydrophilic triptorelin acetate salt by coacervation provide a release period of 1 or 3 months, whereas the more hydrophobic triptorelin pamoate salt microparticles produced by hot-melt extrusion, provides a release period of 6 months. The challenge encountered with the formulation of polymer microparticles is to obtain a sufficiently high drug loading of the compound in the delivery system to be able to administer the dose for a 1- to 6-months treatment within a restricted volume of 1-2 ml. Moreover, it is important to design the system to ensure the release kinetic profiles are correlated to the PLGA degradation rates in vivo based on polymer length and lactide/glycolide ratio. Due to its localized aspect and well-characterized structure, the drug diffusion through the sc. interstitial 
matrix can be precisely described by mathematical models, and used to design drug formulations accordingly [123]. Such models have been used to develop new formulations by understanding the release characteristics from the PLGA microparticles [138]. Moreover, as the sc. administration of small cytotoxic drugs is usually limited by their irritant character, their encapsulation inside micro- or nano-carriers is a route option to increase the injected dose leading to higher local concentration at injection site, without inducing local toxicity [139].

DDS based on drug conjugates were developed to fine-tune the drug release depending on the covalent linker choice between the drug and the polymer. Numerous technologies were developed with PEG-drug conjugates, since PEG is well characterized and already marketed in FDA-approved drug products. Among others we can cite Enzon's PEGylation Linker Technology, Nektar ${ }^{\circledR}$ 's polymer conjugation technology, or TransCon ${ }^{\top M}$ technology. The latter, developed by Ascendis Pharma, enables sustained delivery of small molecules, peptides and proteins [140]. This technology platform was clinically evaluated across multiple therapeutic molecules and indications, ultimately the FDA submission for registration of TransCon hGH is ongoing. The technology is described as a transient binding between the parent drug and a linker-carrier, whose role is to reduce receptor activity and shield it from blood clearance. Following sc. injection, the traceless linker is intended to autohydrolyze and release the unmodified parent drug at a predictable rate. Ascendis ${ }^{T M}$ to date has generated a pipeline of three clinical candidates utilizing TransCon technology, and is currently developing these candidates in the oncology and rare disease endocrinology therapeutic areas [141]. Even though most of the highly concentrated formulations are usually highly viscous, it has to be noted that TransCon technology demonstrated that the polymer-drug conjugates are enabling high drug concentration and low viscosity formulation for sc. injection, and usually decrease local toxicity at the injection site. As discussed above, since the administration of PEG can be related to immunogenic adverse events, alternatives are under investigation to develop non-PEG polymers maintaining its intrinsic advantages (increased half-life, sustained release, increased solubility) and reducing the immunogenicity [142]. Among the few technologies developed based on non-PEG drug conjugates, two are investigating the oncology therapeutic area at preclinical stage. XTEN ${ }^{\circledR}$ technology, developed by Amunix Pharmaceuticals, relies on a proprietary linear polypeptide made of hydrophilic amino acids covalently coupled to a drug substance (peptide, protein or small molecule). XTEN demonstrated to have low immunogenicity while extending the drug substance half-life. Therefore, Amunix has designed the $\mathrm{XPAT}^{\mathrm{TM}}$ platform (XTENylated Protease-Activated T-Cell Engager) based on the well-established and validated XTEN technology as a linker for T-cell engagers in solid tumors. The recombinant structure is composed of the therapeutic molecule (T-cell engager, peptide, protein) linked to the XTEN polypeptide via a proprietary protease release site specific of the targeted tumor environment. This innovative technology is currently under preclinical evaluation (lead optimization stage) and was licensed in January 2020 to Roche for nononcology applications [143]. A second promising technology is being developed by Serina Therapeutics in a Phase II clinical trial for Parkinson therapy and currently under preclinical investigation in oncology. The $\mathrm{POZ}^{\mathrm{TM}}$ drug-delivery platform is based on a water-soluble, low-viscosity polymer poly(2-oxazoline) and can be adapted to proteins, peptides, aptamers or small molecules. The structure of the polymer (linear, branched, pendent) can be designed based on the drug loading required and the terminal site of the polymer can be functionalized to achieve an active targeting of the delivery system. This POZ platform provides a nonimmunogenic delivery system, biodegradable and releasing the drug substance in a sustained manner after SC administration [144,145].

To address the challenge of specific release rates or on-demand release, polymeric implants and microchips were developed for sc. administration [146]. Implants are able to release drug substances over different periods of time, depending on the cancer type and treatment schedule. This technology is already marketed for other indications, for example, as nonbiodegradable birth control implants. In the cancer field, the biodegradable PLGA-based Zoladex ${ }^{\circledR}$ implants were developed about 25 years ago for the delivery of gosereline over 1-3 months, for the treatment of prostate cancer in different settings [147]. One implant was developed by Alza to deliver leuprolide acetate over a period of 12 months at a controlled rate [148]. Also, nonbiodegradable implants are marketed such as Viadur ${ }^{\circledR}$ implant, a $4 \times 45 \mathrm{~mm}$ titanium reservoir, including polyurethane membranes, osmotic tablets and an elastomeric piston to control the drug release over time. More recently, implantable microchips able to store multiple drugs in their most stable form were developed. The drugs are released at the appropriate time and dose by telemetry, either due to a closed loop system based on feedbacks from sensors in the body, or on demand by the patient or the clinician through wireless signals [149,150]. Each microchip contains an array of reservoirs filled either with liquid or solid-phase formulations, according to the stability of the molecules in each of the forms [151]. The first-in-human testing of such wirelessly controlled microchip demonstrated the possibility to obtain equivalent and reproducible daily pharmacokinetic profiles for up to 20 days. The microchip was shown to be biocompatible and well tolerated 
Table 3. Summary of challenges and examples of drug-delivery solutions for subcutaneous administration of anticancer drugs.

\section{Challenge}

Administration of high viscosity, highly concentrated formulations

Sustained drug release

Delivery of combination treatments and on-demand drug release

Example of drug-delivery solution
Organic depot able to self-organize into a
liquid crystal nanostructured matrix
In situ solidifying implant
Peptide self-assembly
PLGA microparticles
Polymer-prodrug

PLGA implant

Controllable, multicompartments, implants

\begin{tabular}{|c|c|}
\hline Example of technology/product available & Ref. \\
\hline FluidCrystal ${ }^{\circledR}$ & [128] \\
\hline Eligard $^{\circledR}$ & [129] \\
\hline Somatuline ${ }^{\circledR}$ Depot & [135] \\
\hline Decapeptyl ${ }^{\circledR}$ SR, Lupron ${ }^{\circledR}$ & {$[148,153]$} \\
\hline $\begin{array}{l}\text { PEG: TransCon }{ }^{T M} \text { (Ascendis Pharma), PEGylation } \\
\text { Linker Technology (ENZON), Polymer } \\
\text { Conjugaison Technology (Nektar) } \\
\text { Non-PEG: XTEN }{ }^{\circledR} \text { (Amunix Pharma), POZ }{ }^{T M} \\
\text { (Serina Therapeutics) }\end{array}$ & {$[141,143,145]$} \\
\hline Zoladex ${ }^{\circledR}$ & [147] \\
\hline
\end{tabular}

Wireless microchips

\begin{tabular}{|c|c|c|c|}
\hline Challenge & Route of administration & Example of technology/product available & Ref. \\
\hline \multirow{3}{*}{$\begin{array}{l}\text { Limited access to parenchymal tissue due to } \\
\text { biological barriers }\end{array}$} & Lung local delivery & Nanoparticle inhalation & {$[160,161]$} \\
\hline & \multirow[t]{2}{*}{ Brain local delivery } & Biodegradable polymer implant Gliadel ${ }^{\circledR}$ & {$[162,163]$} \\
\hline & & CED of nanocarriers & {$[165,166]$} \\
\hline Reverse immune response against tumor cells & Intramuscular, intratumoral or intravenous & Personalized cancer vaccine & {$[170,172]$} \\
\hline
\end{tabular}

CED: Convection-enhanced delivery.

by patients and could potentially be optimized to deliver daily doses of drugs for more than a year. The major challenge remains the very small volume of each reservoir $(<1 \mu \mathrm{l})$, hence requiring very potent drugs or highly concentrated formulations and the nonbiodegradability of the system [152].

\section{Conclusion on sc. delivery of anticancer drugs}

Challenges and examples of drug delivery solutions for the sc. administration of anticancer drugs are summarized in Table 3. Overall, limited efforts were devoted to the potential of sc. administration of small-molecule anticancer drugs, likely because of both local pain at injection site generating tolerance issues [127], and limited volumes of administration requiring highly concentrated formulations and/or highly potent drugs. So far, the main developments were performed for the delivery of hormone agonist or antagonist peptides, for the treatment of hormone-dependent cancer patients, which would require chronic administration. However, this route of administration offers numerous benefits including overall reduced administration costs and might allow for ambulatory treatment and self-administration after patient training therefore increasing patient compliance. It also carries the potential to ultimately implement drug reservoirs that could release drug on demand, or according to an internal dosing schedule. However, as illustrated by the limited number of newly approved sustained-release formulations for sc. administration of cancer drugs since 2005, progression to the market for these formulations still remains a major challenge.

\section{Alternative drug delivery routes}

Oral, iv. and sc. routes of administration are the most widely developed routes in the field of oncology DDS. However, some alternative DDS were designed to be administered through a specific route to answer a precise challenge or to reach a specific organ and are summarized in Table 4.

As an example, pulmonary delivery via inhalation is one way to target lung cancer or lung metastases. Indeed, inhalation enables the local delivery of high drug doses while reducing the potential systemic side effects due to low diffusion of the drug in blood circulation. Inhaled chemotherapy was developed with drugs such as doxorubicin [154], cisplatin [155], carboplatin [156] in clinical trials and was recognized as feasible in most of these trials. However, studies failed in Phase II at best due to several limitations of the delivery route. First of all, the high drug potency combined with the local deposition of the drug in the lungs led to local severe toxicity $[157,158]$. Moreover, the massive 
loss of drug in the pulmonary tract required the administration of high doses, therefore inducing long inhalation periods to deliver the right dose. Due to a low benefit/risk ratio, a high financial cost and the need for a specialized device, inhalation as a route of administration is not the most widely recommended route for chemotherapies. However, some early trials are ongoing to address the challenges described above. As such, liposomes or solid lipid nanoparticles showed a reduced local toxicity due to the protection of the drug inside the carrier [159], and a potential modification of the drug release or a specific cell targeting via ligands covalently linked to the nanocarrier [160,161].

Local drug delivery was also developed for brain tumor treatment with the direct implantation in the tumor resection cavity of a marketed biodegradable wafer, Gliadel ${ }^{\circledR}$. The Gliadel implant is able to release the cytotoxic small-molecule carmustin and was approved in 1996 as an adjunct to surgery to prolong survival in patients with recurrent glioblastoma multiform [162]. However, since transport of the drug substance in the brain parenchyma relies on diffusion, clinical benefit of Gliadel was limited [163]. Other biodegradable polymeric delivery technologies were explored besides macroscopic wafer technologies. Biodegradable copolymer PLGA microspheres, loaded with antitumor therapies can be administered by stereotaxic, computer-assisted approaches to brain areas in a very precise manner. Their small size enables multiple and spatially distributed implantations in the walls of the tumor cavity, compared with a macroscopic wafer. This strategy was evaluated in an open-label Phase II study using microspheres loaded with the radio-sensitizing 5-FU, after tumor resection followed by radiotherapy. No significant difference in median overall survival was observed, likely due to a poor design and insufficient power of the study to be able to demonstrate the potential increase in survival further to the diffusion and the implantation of 5-FU-loaded microspheres in the wall of the cavity resection [164]. Convection-enhanced delivery using hydrostatic pressure to drive the drug deeper into the tumor tissue has attempted to overcome this challenge [165]. Recent clinical trials showed that convection-enhanced delivery is safe and feasible, and this technique was recently coupled to the formulation of drugs into nanoparticles, providing the optimized nanoformulation in terms of size and surface properties to be able to circulate through brain pores [166].

Finally, in the field of immuno-oncology, the therapeutic cancer vaccination concept was developed over the past 20 years [167]. Therapeutic cancer vaccination is based on the administration of tumor-associated antigen combined with an adjuvant to modify the T-cell ignorance and reverse the immune response against the tumor cells $[168,169]$. These vaccines are usually administered via parenteral routes, for example, intramuscular, sc. or intramuscular. Intramuscular injection is the most common one due the simple injection and the low local reactions that can be observed compared with intradermal. Despite the successful early clinical data, most of the clinical trials in cancer vaccines failed in Phase I/II due to insufficient therapeutic efficacy. One of the most advanced systems currently developed for the intracellular delivery of biomacromolecules are lipid-based nanoparticles, exploited by Moderna Therapeutics Inc. to deliver mRNA vaccines and increase the therapeutic efficacy of the vaccines. The developed vaccines are administered either through intramuscular or direct intratumoral injection. After demonstrating the initiation of a strong innate immune response in a rhesus macaque model [170], Moderna launched clinical trial Phase II study using mRNA-4157 loaded lipid-based nanoparticles, for the treatment of multiple types of solid tumors and melanoma [171] and recently a Phase I study on mRNA-5671 ro colorectal cancer, lung cancer and pancreatic cancer. Similarly, BioNTech SE is clinically evaluating their FixVac ${ }^{\circledR}$ technology platform. The drug candidates are RNA lipoplexes comprising at least one cationic lipid and a combination of unmodified, pharmacologically optimized mRNA, encoding known cancer-specific shared antigens. Organ-specific mRNA delivery aims to ensure that the active proteins are produced exclusively in tissues and cells of interest. Multiple candidates are currently under clinical trials Phase I and II for several cancer indications: melanoma, head and neck cancer, prostate cancer, breast cancer and other solid tumors [172,173].

\section{Conclusion}

Increasing the understanding of cancer pathology and development of novel therapeutic approaches have led to the idea that cancer is not only one but many diseases even within one single indication, that need to be tackled with well-defined and rationalized cancer treatments. DDS have a major role to play in the efficient administration of anticancer drugs orally, intravenously or subcutaneously. The development of innovative formulations has already proven to significantly improve efficacy and toxicity profiles of many therapeutic modalities, as described in this review.

Key challenges and opportunities for each delivery route discussed above are summarized in Figure 2. In oral delivery of cancer drugs, enhancing the bioavailability and decreasing inter- and intra-patient variability will likely remain key challenges. We consider that a better understanding of GI barriers crossing mechanisms at different 


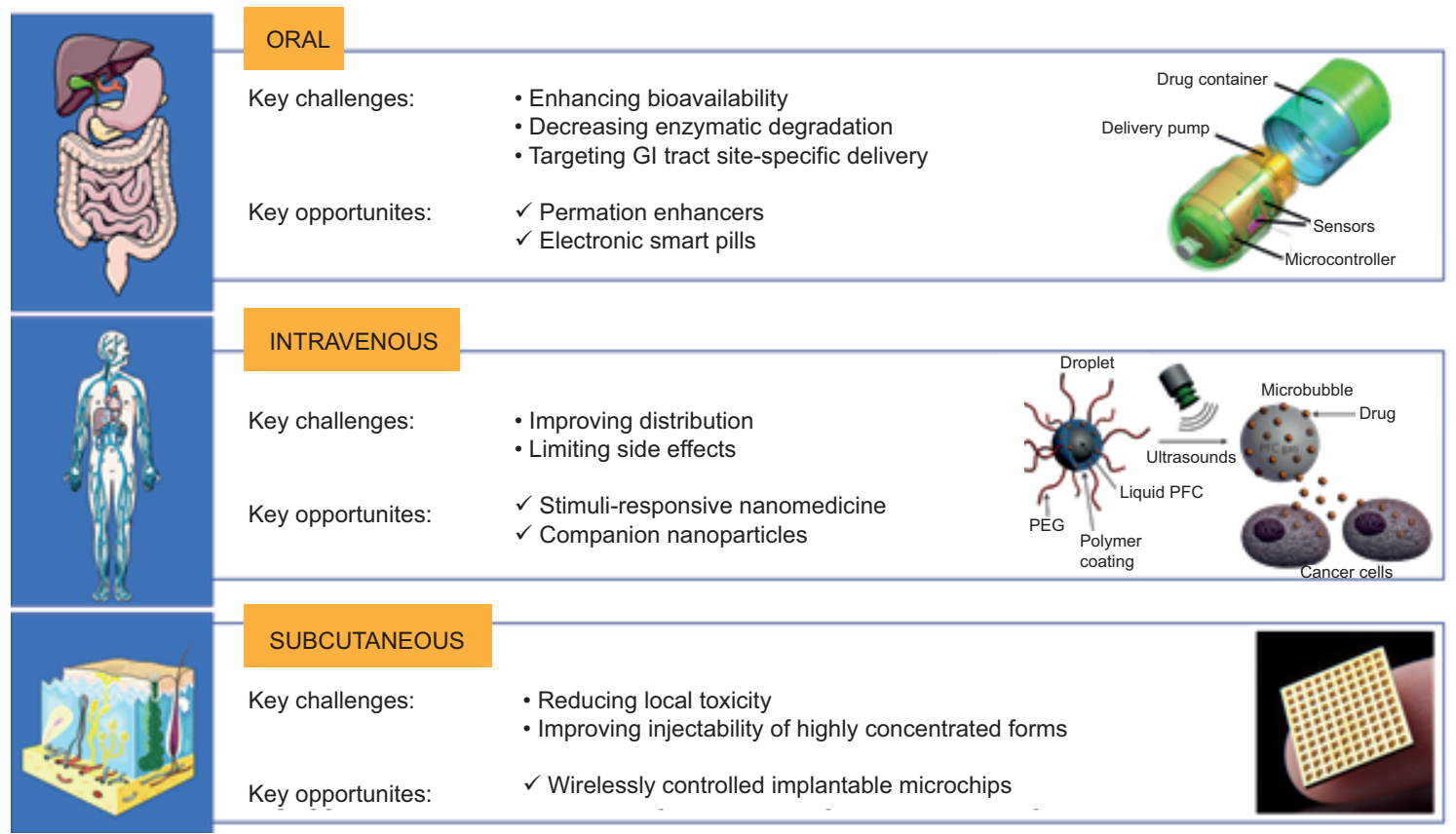

Figure 2. Key challenges and opportunities for each delivery route for the coming years. Digestive track system, venous system and subcutis are from Servier Medical Art, under the terms of the license Creative Commons Attribution 3.0, France.

Electronic pill scheme is from www.medimetrics.com, stimuli-responsive nanomedicine scheme is reproduced with permission from [106] and implantable microchips picture is from microchipsbiotech.com.

delivery sites along the GI tract would allow to fine-tune delivery systems, according to the physicochemical characteristics of the drug and the considered indication. Moreover, innovative technologies for oral delivery need to take into account the low bioavailability of the drug and therefore the need to deliver high doses of biologics or small molecules regularly. iv. administration has been the most explored delivery route to implement personalized treatment schemes using more challenging molecules such as biologics, or innovative nano-DDS. This delivery route will likely remain among the most preferred administration route, ensuring the best control over bioavailability, although challenges associated with drug distribution need to be addressed to significantly improve patient quality of life. Finally, the administration of chemotherapies via SC administration still does not encounter much momentum as an appropriate delivery route for anticancer treatments with only one FDA approved drug since 2013, Xgeva ${ }^{\circledR}$ from Amgen. As stated above, the main innovative strategies for sc. delivery are the development of polymer-prodrugs to develop highly concentrated formulations that upon sc. injection form a depot for drug sustained and controlled release.

\section{Future perspective}

Regarding the evolution of the field in the next decades, the oral delivery route is already encountering a renewed interest with the development of cancer drugs with wider therapeutic windows, as well as the advances in 3Dprinting and personalized delivery digital devices.

iv. route is continuously exploring new technologies toward personalized medicine, especially in the field of nanotechnologies. To our knowledge, efforts are expected to be focused on the development of optimized nanomedicines and their industrialization, in which versatility offers the possibility to tackle the different challenges associated with cancer diversity, from patient selection to therapeutic response follow-up. From a long-term perspective, significant progress in the development of sc. implantable microchips that could deliver drugs on demand through closed loop systems or wireless control, will likely make pharmaceutical companies reconsider this administration route for future treatment schemes.

Overall, increasing understanding of cancer progression and resistance mechanisms and continuous development of innovative DDS make the outlook for anticancer drugs delivery promising. We believe that the close collaboration 
between drug delivery scientists, biologists and clinical oncologists is a key enabler to pursue the implementation of personalized therapies. This should allow developing the most appropriate DDS that is optimized to suit each therapeutic drug and to ensure a major clinical benefit for the patient through delivery of the right drug at the right place and over the right period of time while reducing off-target toxicity.

\section{Executive summary}

\section{Current challenges in anticancer drug delivery}

- Challenges associated with the delivery of cancer treatments are the multiplicity of available drugs and their associated physicochemical properties, the safety of the administration, the potential difficulty to reach a specific tumor location and the complexity of treatment schemes.

- The development of a drug-delivery strategy and the choice of administration route should consider altogether the aforementioned challenges.

Oral administration

- Oral route is the patients' preferred mode of delivery due to simplicity and noninvasiveness.

- This route faces two major challenges: the low bioavailability of the drug substance (solubility and/or permeability issues depending on the molecule) and the targeted delivery to a defined section of the GI tract.

- The main innovative strategies include drug nanonization, lipid-based formulations, permeation enhancers and gastroretentive dosage forms.

- Oral route is commonly used in multiple cancer indications and is perfectly appropriate for gastrointestinal cancers. It also provides a specific entry into the lymphatic system relevant in lymphoma and some metastatic cancers.

- More recent modalities of treatment such as noncytotoxic targeted agents, immunotherapy or hormonal therapy, characterized by a wider therapeutic window, may benefit from novel oral formulations.

Intravenous administration

- Intravenous administration is the most commonly route for cancer drugs, ensuring high bioavailability and low inter-/intra-patient variability.

- This delivery route faces numerous challenges such as: non-favorable pharmacokinetics parameters of the drug substance, nonspecificity of some chemotherapeutic agents leading to severe side effects (off- and on-target toxicity) and difficulty to overcome highly protective barriers (e.g., the blood-brain barrier).

- The main innovative strategies are currently based on the use of nanomedicines.

- Initially developed to deliver cytotoxic small molecules, rational design of the nanocarriers should extend their use to the delivery of biomolecules (peptides, proteins or RNA/DNA).

\section{Subcutaneous administration}

- Strong efforts have been devoted to the subcutaneous administration of anticancer drugs but very few approvals have been so far registered.

- This delivery route faces two major challenges: the administration of sustained doses over longer period of time, and the control of multiple release rates.

- The main innovative strategies are the polymer-prodrugs systems enabling highly concentrated formulations to form a subcutaneous depot for drug-controlled release. Some of the developed technologies will enable a significant decrease of the viscosity and facilitate injectability.

- More ambitious and complex technologies such as implantable microchips will be able to deliver drugs on demand through closed loop systems or wireless control and polymer-prodrugs bioconjugates for drug-controlled release and reduced local toxicities.

\section{Financial \& competing interests disclosure}

All the authors are or were employees of Ipsen, a pharmaceutical company that markets oncology products at the time of paper writing. The authors have no other relevant affiliations or financial involvement with any organization or entity with financial interest in or financial conflict with the subject matter or materials discussed in the manuscript apart from those disclosed.

No writing assistance was utilized in the production of this manuscript.

\section{Open access}

This work is licensed under the Attribution-NonCommercial-NoDerivatives 4.0 Unported License. To view a copy of this license, visit http://creativecommons.org/licenses/by-nc-nd/4.0/

\section{References}

Papers of special note have been highlighted as: $\bullet$ of interest; $\bullet \bullet$ of considerable interest 
1. World Health Organization website. Health Topics - Cancer - Overview. www.who.int/cancer/en/

2. Center Watch. FDA approved drugs for oncology. www.centerwatch.com/drug-information/fda-approved-drugs/therapeutic-area/12/oncology

3. National Cancer Institute training module. Cancer Registration \& Surveillance Modules - Cancer Treatment. https://training.seer.cancer.gov/treatment/

4. Subklewe M, Von Bergwelt-Baildon M, Humpe A. Chimeric antigen receptor T cells: a race to revolutionize cancer therapy. Transfus. Med. Hemotherapy 46(1), 15-24 (2019).

5. Lewis AL, Richard J. Challenges in the delivery of peptide drugs: an industry perspective. Ther. Deliv. 6(2), 149-163 (2015).

6. Richard J. Parenteral Biologics Delivery: recent progresses, key challenges and perspectives. Eur. J. Parenter. Pharm. Sci. 17(3), 94-109 (2012).

7. Collins DS, Sánchez-Félix M, Badkar AV, Mrsny R. Accelerating the development of novel technologies and tools for the subcutaneous delivery of biotherapeutics. J. Control. Rel. 321, 475-482 (2020).

8. Thanki K, Gangwal RP, Sangamwar AT, Jain S. Oral delivery of anticancer drugs: challenges and opportunities. J. Control. Rel. 170(1), $15-40$ (2013).

9. Junyaprasert VB, Morakul B. Nanocrystals for enhancement of oral bioavailability of poorly water-soluble drugs. Asian J. Pharm. Sci. 10(1), 13-23 (2015).

10. Rabinow BE. Nanosuspensions in drug delivery. Nat. Rev. Drug Discov. 3(9), 785-796 (2004).

11. Junghanns JUAH, Müller RH. Nanocrystal technology, drug delivery and clinical applications. Int. J. Nanomedicine 3, 295-309 (2008).

12. Wu Y, Loper A, Landis $\mathrm{E}$ et al. The role of biopharmaceutics in the development of a clinical nanoparticle formulation of MK-0869: a Beagle dog model predicts improved bioavailability and diminished food effect on absorption in human. Int. J. Pharm. 285(1-2), 135-146 (2004).

13. Porter CJH, Trevaskis NL, Charman WN. Lipids and lipid-based formulations: optimizing the oral delivery of lipophilic drugs. Nat. Rev. Drug Discov. 6(3), 231-248 (2007).

14. Jannin V, Musakhanian J, Marchaud D. Approaches for the development of solid and semi-solid lipid-based formulations. Adv. Drug Deliv. Rev. 60(6), 734-746 (2008).

15. Griesser J, Hetényi G, Moser M, Demarne F, Jannin V, Bernkop-Schnürch A. Hydrophobic ion pairing: key to highly payloaded self-emulsifying peptide drug delivery systems. Int. J. Pharm. 520(1-2), 267-274 (2017).

16. Aungst BJ. Intestinal permeation enhancers. J. Pharm. Sci. 89(4), 429-442 (2000).

17. Maher S, Mrsny RJ, Brayden DJ. Intestinal permeation enhancers for oral peptide delivery. Adv. Drug Deliv. Rev. 106, 277-319 (2016).

18. Clinical trials. Octreotide capsules. https://clinicaltrials.gov/ct2/show/NCT03252353

19. Twarog C, Fattah S, Heade J, Maher S, Fattal E, Brayden DJ. Intestinal permeation enhancers for oral delivery of macromolecules: a comparison between salcaprozate sodium (SNAC) and sodium caprate (c10). Pharmaceutics 11(2), 1-21 (2019).

- Recent review comparing two strategies for the delivery of biologics with permeation enhancers. This DDS is widely explored currently in clinical phases.

20. Davies M, Pieber TR, Hartoft-Nielsen ML, Hansen OKH, Jabbour S, Rosenstock J. Effect of oral semaglutide compared with placebo and subcutaneous semaglutide on glycemic control in patients with type 2 diabetes a randomized clinical trial. J. Am. Med. Assoc. 318(15), 1460-1470 (2017).

21. Richard J. Challenges in oral peptide delivery: lessons learnt from the clinic and fututre prospects. Ther. Deliv. 7(2), 117-138 (2016).

22. Date AA, Hanes J, Ensign LM. Nanoparticles for oral delivery: design, evaluation and state-of-the-art. J. Control. Rel. 240, 504-526 (2016).

23. Duncan R. Polymer conjugates as anticancer nanomedicines. Nat. Rev. Cancer 6(9), 688-701 (2006).

24. Benny O, Fainaru O, Adini A et al. An orally delivered small-molecule formulation with antiangiogenic and anticancer activity. 46(2), 220-231 (2010).

25. Khafagy ES, Morishita M. Oral biodrug delivery using cell-penetrating peptide. Adv. Drug Deliv. Rev. 64(6), 531-539 (2012).

26. Khafagy ES, Morishita M, Ida N, Nishio R, Isowa K, Takayama K. Structural requirements of penetratin absorption enhancement efficiency for insulin delivery. J. Control. Rel. 143(3), 302-310 (2010).

27. Nielsen EJB, Yoshida S, Kamei $\mathrm{N}$ et al. In vivo proof of concept of oral insulin delivery based on a co-administration strategy with the cell-penetrating peptide penetratin. J. Control. Rel. 189, 19-24 (2014).

28. Kamei N, Kikuchi S, Takeda-Morishita M et al. Determination of the optimal cell-penetrating peptide sequence for intestinal insulin delivery based on molecular orbital analysis with self-organizing maps. J. Pharm. Sci. 102(2), 469-479 (2013).

29. Couvreur P, Stella B, Harivardhan Reddy L et al. Squalenoyl nanomedicines as potential therapeutics. Nano Lett. 6(11), 2544-2548 (2006). 
30. Kotelevets L, Chastre E, Caron J et al. A squalene-based nanomedicine for oral treatment of colon cancer. Cancer Res. 77(11), 2964-2975 (2017).

31. Ensign LM, Cone R, Hanes J. Oral drug delivery with polymeric nanoparticles: the gastrointestinal mucus barriers. Adv. Drug Deliv. Rev. 64(6), 557-570 (2012).

32. Lai SK, Wang YY, Hanes J. Mucus-penetrating nanoparticles for drug and gene delivery to mucosal tissues. Adv. Drug Deliv. Rev. 61(2), 158-171 (2009).

33. Lollo G, Gonzalez-Paredes A, Garcia-Fuentes M, Calvo P, Torres D, Alonso MJ. Polyarginine nanocapsules as a potential oral peptide delivery carrier. J. Pharm. Sci. 106(2), 611-618 (2017).

34. Rapamycin Holdings I DBA EB. NCT04375813: trial of encapsulated rapamycin (eRAPA) for bladder cancer prevention. Clinical Trials.gouv https://clinicaltrials.gov/ct2/show/NCT0 4375813

35. Bohan PMK, Cindass JL, Chick RC et al. Results of a Phase Ib trial of encapsulated rapamycin in prostate cancer patients under active surveillance to prevent progression. J. Clin. Oncol. 38(Suppl. 5), 34-34 (2020).

36. Imran M. A novel approach to the oral delivery of biologics, peptides and antibodies. (2016). www.ondrugdelivery.com

37. RaniTherapeutics. RaniPill - first in human. https://clinicaltrials.gov/ct2/show/NCT03798912? term=ranipill\&draw $=2 \&$ rank=1

38. Abramson A, Caffarel-salvador E, Khang M et al. An ingestible self-orienting system for oral delivery of macromolecules. Science 363 , 611-615 (2019).

39. Lopes CM, Bettencourt C, Rossi A, Buttini F, Barata P. Overview on gastroretentive drug delivery systems for improving drug bioavailability. Int. J. Pharm. 510(1), 144-158 (2016).

40. Bellinger AM, Jafari M, Grant TM et al. Oral, ultra-long-lasting drug delivery: application toward malaria elimination goals. Sci. Transl. Med. 8(365), 365ra157-365ra157 (2016).

41. Liu J, Pang Y, Zhang S et al. Triggerable tough hydrogels for gastric resident dosage forms. Nat. Commun. 8(1), 1-9 (2017).

42. Liu L, Yao WD, Rao YF, Lu XY, Gao JQ. pH-responsive carriers for oral drug delivery: challenges and opportunities of current platforms. Drug Deliv. 24(1), 569-581 (2017).

43. Thakral S, Thakral NK, Majumdar DK. Eudragit ${ }^{\circledR}$ : a technology evaluation. Expert Opin. Drug Deliv. 10(1), 131-149 (2012).

44. Trevaskis NL, Kaminskas LM, Porter CJH. From sewer to saviour-targeting the lymphatic system to promote drug exposure and activity. Nat. Rev. Drug Discov. 14(11), 781-803 (2015).

45. Chaudhary S, Garg T, Murthy RSR, Rath G, Goyal AK. Recent approaches of lipid-based delivery system for lymphatic targeting via oral route. J. Drug Target. 22(10), 871-882 (2014).

46. Banerjee A, Pathak S, Subramanium VD, Dharanivasan G, Murugesan R, Verma RS. Strategies for targeted drug delivery in treatment of colon cancer: current trends and future perspectives. Drug Discov. Today 22(8), 1224-1232 (2017).

47. Esseku F, Adeyeye MC. Bacteria and pH-sensitive polysaccharide-polymer films for colon targeted delivery. Crit. Rev. Ther. Drug Carr. Syst. 28(5), 395-445 (2012).

48. Sinha VR, Mittal BR, Bhutani KK, Kumria R. Colonic drug delivery of 5-fluorouracil: an in vitro evaluation. Int. J. Pharm. 269(1), 101-108 (2004).

49. Soppimath KS, Kulkarni AR, Aminabhavi TM. Controlled release of antihypertensive drug from the interpenetrating network poly(vinyl alcohol)-guar gum hydrogel microspheres. J. Biomater. Sci. Polym. Ed. 11(1), 27-43 (2000).

50. Palo M, Holländer J, Suominen J, Yliruusi J, Sandler N. 3D printed drug delivery devices: perspectives and technical challenges. Expert Rev. Med. Devices 14(9), 685-696 (2017).

51. US FDA. Spritam. www.accessdata.fda.gov/drugsatfda_docs/nda/2015/207958Orig1s000TOC.cfm

52. Becker $\mathrm{D}$, Zhang J, Heimbach $\mathrm{T}$ et al. Novel orally swallowable IntelliCap ${ }^{\circledR}$ device to quantify regional drug absorption in human GI tract using diltiazem as model drug. AAPS PharmSciTech 15(6), 1490-1497 (2014).

53. Mazzaferro S, Bouchemal K, Ponchel G. Oral delivery of anticancer drugs I: general considerations. Drug Discov. Today 18(1-2), 25-34 (2013).

54. Brayden DJ, Hill TA, Fairlie DP, Maher S, Mrsny RJ. Systemic delivery of peptides by the oral route: formulation and medicinal chemistry approaches. Adv. Drug Deliv. Rev. doi:10.1016/j.addr.2020.05.007 (2020) (Epub ahead of print).

55. O'neill VJ, Twelves CJ. Oral cancer treatment: developments in chemotherapy and beyond. Br. J. Cancer 87(9), $933-937$ (2002).

56. Huda S, Alam A, Sharma PK. Smart nanocarriers-based drug delivery for cancer therapy: an innovative and developing strategy. J. Drug Deliv. Sci. Technol. 60, 102018 (2020).

57. Shi J, Kantoff PW, Wooster R, Farokhzad OC. Cancer nanomedicine: progress, challenges and opportunities. Nat. Rev. Cancer 17, 20-37 (2016).

58. Gref R, Minamitake Y, Peracchia MT, Trubetskoy V, Torchilin V, Langer R. Biodegradable long-circulating polymeric nanospheres. Science 263(5153), 1600-1603 (1994). 
59. Klibanov AL, Maruyama K, Torchilin VP, Huang L. Amphipathic polyethyleneglycols effectively prolong the circulation time of liposomes. FEBS Lett. 268(1), 235-237 (1990).

60. Barenholz Y. Doxil - The first FDA-approved nano-drug: lessons learned. J. Control. Rel. 160(2), 117-134 (2012).

61. Zhang P, Sun F, Liu S, Jiang S. Anti-PEG antibodies in the clinic: current issues and beyond PEGylation. J. Control. Rel. 244, 184-193 (2016).

62. Yang Q, Jacobs TM, McCallen JD et al. Analysis of pre-existing IgG and IgM antibodies against polyethylene glycol (PEG) in the general population. Anal. Chem. 88(23), 11804-11812 (2016).

63. Knop K, Hoogenboom R, Fischer D, Schubert US. Poly(ethylene glycol) in drug delivery: pros and cons as well as potential alternatives. Angew. Chemie - Int. Ed. 49(36), 6288-6308 (2010).

64. Shiraishi K, Yokoyama M. Toxicity and immunogenicity concerns related to PEGylated-micelle carrier systems: a review. Sci. Technol. Adv. Mater. 20(1), 324-336 (2019).

65. McSweeney MD, Versfeld ZC, Carpenter DM, Lai SK. Physician awareness of immune responses to polyethylene glycol-drug conjugates. Clin. Transl. Sci. 11(2), 162-165 (2018).

66. Anchordoquy TJ, Simberg D. Watching the gorilla and questioning delivery dogma. J. Control. Rel. 262, 87-90 (2017).

67. Deng Y, Saucier-Sawyer JK, Hoimes CJ et al. The effect of hyperbranched polyglycerol coatings on drug delivery using degradable polymer nanoparticles. Biomaterials 35(24), 6595-6602 (2014).

68. Zhang T, She Z, Huang Z, Li J, Luo X, Deng Y. Application of sialic acid/polysialic acid in the drug delivery systems. Asian J. Pharm. Sci. 9(2), 75-81 (2014).

69. Metselaar JM, Bruin P, De Boer LWT et al. A novel family of L-amino acid-based biodegradable polymer-lipid conjugates for the development of long-circulating liposomes with effective drug-targeting capacity. Bioconjug. Chem. 14(6), 1156-1164 (2003).

70. Thi TTH, Pilkington EH, Nguyen DH, Lee JS, Park KD, Truong NP. The importance of poly (ethylene glycol) alternatives for overcoming PEG immunogenicity in drug. Polymers (Basel). 12(298), (2020). www.mdpi.com/2073-4360/12/2/298\#cite

-. Despite the efficiency of PEG to provide stealth properties to nanoparticles, this paper highlights the need to find alternatives with better safety profile.

71. Bertrand N, Wu J, Xu X, Kamaly N, Farokhzad OC. Cancer nanotechnology: the impact of passive and active targeting in the era of modern cancer biology. Adv. Drug Deliv. Rev. 66, 2-25 (2014).

72. Maeda $H$. The enhanced permeability and retention (EPR) effect in tumor vasculature: the key role of tumor-selective macromolecular drug targeting. Adv. Enzyme Regul. 41(1), 189-207 (2001).

73. Kratz F. Albumin as a drug carrier: design of prodrugs, drug conjugates and nanoparticles. J. Control. Rel. 132(3), 171-183 (2008).

74. Maeda H. Toward a full understanding of the EPR effect in primary and metastatic tumors as well as issues related to its heterogeneity. Adv. Drug Deliv. Rev. 91, 3-6 (2015).

75. Miller MA, Gadde S, Pfirschke C et al. Predicting therapeutic nanoparticle efficacy using a companion MR imaging nanoparticle. Sci. Transl. Med. 7(314), 314ra183 (2015).

76. Arrieta O, Medina LA, Estrada-Lobato E, Ramírez-Tirado LA, Mendoza-García VO, De La Garza-Salazar J. High liposomal doxorubicin tumour tissue distribution, as determined by radiopharmaceutical labelling with $99 \mathrm{mTc}-\mathrm{LD}$, is associated with the response and survival of patients with unresectable pleural mesothelioma treated with a combination of liposomal do. Cancer Chemother. Pharmacol. 74(1), 211-215 (2014).

77. Yokoi K, Tanei T, Godin B et al. Serum biomarkers for personalization of nanotherapeutics-based therapy in different tumor and organ microenvironments. Cancer Lett. 345(1), 48-55 (2014).

78. Vaz J, Ansari D, Sasor A, Andersson R. SPARC: a potential prognostic and therapeutic target in pancreatic cancer. Pancreas 44(7), 1024-1035 (2015).

79. Giordano G, Pancione M, Olivieri N et al. Nano albumin bound-paclitaxel in pancreatic cancer: current evidences and future directions. World J. Gastroenterol. 23(32), 5875-5886 (2017).

80. Munsell EV, Ross NL, Sullivan MO. Journey to the center of the cell: current nanocarrier design strategies targeting biopharmaceuticals to the cytoplasm and nucleus. Curr. Pharm. Des. 22(9), 1227-1244 (2016).

81. Heath TD, Fraley RT, Papahadjopoulos D. Antibody targeting of liposomes: cell specificity obtained by conjugation of F(ab') 2 to vesicle surface. Science (80-.). 210(4469), 539-541 (1980).

82. Von Hoff DD, Mita MM, Ramanathan RK et al. Phase I study of PSMA-targeted docetaxel-containing nanoparticle BIND-014 in patients with advanced solid tumors. Clin. Cancer Res. 22(13), 3157-3163 (2016).

83. Autio KA, Dreicer R, Anderson J et al. Safety and efficacy of BIND-014, a docetaxel nanoparticle targeting prostate-specific membrane antigen for patients with metastatic castration-resistant prostate cancer: a Phase II clinical trial. JAMA Oncol. 4(10), 1344-1351 (2018).

- Good example of an innovative nanotechnology system for the targeted delivery of anticancer drug, currently in clinical phase.

84. Xu L, Tang W-H, Huang C-C et al. Systemic p 53 gene therapy of cancer with immunolipoplexes targeted by anti-transferrin receptor scFv. Mol. Med. 7(10), 723-734 (2018).

85. Pardridge WM. The blood-brain barrier: bottleneck in brain drug development. NeuroRx 2(1), 3-14 (2005). 
86. Rautio J, Laine K, Gynther M, Savolainen J. Prodrug approaches for CNS delivery. AAPS J. 10(1), 92-102 (2008).

87. Gao H, Pang Z, Jiang X. Targeted delivery of nano-therapeutics for major disorders of the central nervous system. Pharm. Res. 30(10), 2485-2498 (2013).

88. Zhou Y, Peng Z, Seven ES, Leblanc RM. Crossing the blood-brain barrier with nanoparticles. J. Control. Rel. 270, 290-303 (2018).

89. Chithrani BD, Ghazani AA, Chan WCW. Determining the size and shape dependence of gold nanoparticle uptake into mammalian cells. Nano Lett. 6(4), 662-668 (2006).

90. De Jong WH, Hagens WI, Krystek P, Burger MC, Sips AJ A M, Geertsma RE. Particle size-dependent organ distribution of gold nanoparticles after intravenous administration. Biomaterials 29(12), 1912-1919 (2008).

91. Hsiao PF, Tsai HC, Peng S et al. Transdermal delivery of poly(ethylene glycol)-co-oleylamine modified gold nanoparticles: effect of size and shape. Mater. Chem. Phys. 224, 22-28 (2019).

92. Lalegani Z, Seyyed Ebrahimi SA. Optimization of synthesis for shape and size controlled silver nanoparticles using response surface methodology. Colloids Surfaces A Physicochem. Eng. Asp. 595, 124647 (2020).

93. Murugan K, Choonara YE, Kumar P, du Toit LC, Pillay V. Cellular internalisation kinetics and cytotoxic properties of statistically designed and optimised neo-geometric copper nanocrystals. Mater. Sci. Eng. C 78, 376-388 (2017).

94. Zhang Z, Liu C, Li C, Wu W, Jiang X. Shape effects of cylindrical versus spherical unimolecular polymer nanomaterials on in vitro and in vivo behaviors. Research 2019, 1-13 (2019).

95. Visaveliya NR, Köhler JM. Single-step in situ assembling routes for the shape control of polymer nanoparticles. Biomacromolecules 19(3), 1047-1064 (2018).

96. Gratton SEA, Pohlhaus PD, Lee J, Guo J, Cho MJ, DeSimone JM. Nanofabricated particles for engineered drug therapies: a preliminary biodistribution study of PRINT ${ }^{\mathrm{TM}}$ nanoparticles. J. Control. Rel. 121(1-2), 10-18 (2007).

97. Merkel TJ, Chen K, Jones SW et al. The effect of particle size on the biodistribution of low-modulus hydrogel PRINT particles. J. Control. Rel. 162(1), 37-44 (2012).

98. Dumont EF, Oliver AJ, Ioannou C et al. A novel inhaled dry-powder formulation of ribavirin allows for efficient lung delivery in healthy participants and those with chronic obstructive pulmonary disease in a Phase I study. Antimicrob. Agents Chemother. 64(5), 1-15 (2020).

99. Xiao Y, Shi K, Qu Y, Chu B, Qian Z. Engineering nanoparticles for targeted delivery of nucleic acid therapeutics in tumor. Mol. Ther. Methods Clin. Dev. 12, 1-18 (2019).

100. Kullberg M, Mccarthy R, Anchordoquy TJ. Systemic tumor-specific gene delivery. J. Control. Rel. 172, 730-736 (2013).

101. Shirley JL, De Jong YP, Terhorst C, Herzog RW. Immune responses to viral gene therapy vectors. Mol. Ther. 28(3), 709-722 (2020).

102. Hinderer C, Katz N, Buza EL et al. Severe toxicity in nonhuman primates and piglets following high-dose intravenous administration of an adeno-associated virus vector expressing human SMN. 29(3), 285-298 (2018).

103. Wong KR, Menendez E, Craik CS, Kavanaugh WM, Vasiljeva O. In vivo imaging of protease activity by probody therapeutic activation. Biochimie 122, 62-67 (2016).

104. Polu KR, Lowman HB. Probody therapeutics for targeting antibodies to diseased tissue. Expert Opin. Biol. Ther. 14(8), 1049-1053 (2014).

105. Lin J, Sagert J. Innovations for next-generation antibody-drug conjugates. In: Cancer Drug Discovery and Development 281-298 (2018).

106. Mura S, Nicolas J, Couvreur P. Stimuli-responsive nanocarriers for drug delivery. Nat. Mater. 12(11), 991-1003 (2013).

-. Describes the opportunities in preclinical and clinical stage for the targeted delivery using stimuli-responsive drug delivery systems.

107. Clinical trials. Thermodox. https://clinicaltrials.gov/ct2/show/NCT00617981

108. Lyon PC, Griffiths LF, Lee J et al. Clinical trial protocol for TARDOX: a Phase I study to investigate the feasibility of targeted release of lyso-thermosensitive liposomal doxorubicin (ThermoDox $\left.{ }^{\circledR}\right)$ using focused ultrasound in patients with liver tumours. J. Ther. Ultrasound 5(1), 1-8 (2017).

109. Poon C, McMahon D, Hynynen K. Noninvasive and targeted delivery of therapeutics to the brain using focused ultrasound. Neuropharmacology 120, 20-37 (2017).

110. Lammers PT, Gremse F, Lammers T et al. Theranostic USPIO-loaded microbubbles for mediating and monitoring blood-brain barrier permeation. Adv. Funct. Mater. 25(1), 36-43 (2015).

111. Huang HY, Liu HL, Hsu PH et al. A multitheragnostic nanobubble system to induce blood-brain barrier disruption with magnetically guided focused ultrasound. Adv. Mater. 27(4), 655-661 (2015).

112. McMahon D, Hynynen K. Acute inflammatory response following increased blood-brain barrier permeability induced by focused ultrasound is dependent on microbubble dose. Theranostics 7(16), 3989-4000 (2017).

113. Tsai HC, Tsai CH, Chen WS, Inserra C, Wei KC, Liu HL. Safety evaluation of frequent application of microbubble-enhanced focused ultrasound blood-brain-barrier opening. Sci. Rep. 8(1), 1-13 (2018).

114. Zhang RX, Wong HL, Xue HY, Eoh JY, Wu XY. Nanomedicine of synergistic drug combinations for cancer therapy - strategies and perspectives. J. Control. Rel. 240, 489-503 (2016). 
115. Cortes JE, Goldberg SL, Feldman EJ et al. Phase II, multicenter, randomized trial of CPX-351 (cytarabine:daunorubicin) liposome injection versus intensive salvage therapy in adults with first relapse AML. Cancer2 121(2), 234-242 (15AD).

116. Fani M, Nicolas GP, Wild D. Somatostatin receptor antagonists for imaging and therapy. J. Nucl. Med. 58(Suppl. 2), S61-S66 (2017).

117. US FDA. Luthatera. www.fda.gov/NewsEvents/Newsroom/PressAnnouncements/ucm594043.htm.

118. Strosberg J, El-Haddad G, Wolin E et al. Phase III trial of 177 Lu-dotatate for midgut neuroendocrine tumors. N. Engl. J. Med. 376(2), $125-135$ (2017).

119. Li Y, Lin TY, Luo Y et al. A smart and versatile theranostic nanomedicine platform based on nanoporphyrin. Nat. Commun. 5(4712), (2014).

120. Paliwal R, Babu RJ, Palakurthi S. Nanomedicine scale-up technologies: feasibilities and challenges. AAPS PharmSciTech 15(6), 1527-1534 (2014)

121. Tyner K, Sadrieh N. Considerations when submitting nanotherapeutics to FDA/CDER for regulatory review. Methods Mol. Biol. 697 , 17-31 (2011).

-. Very useful overview of the complex regulatory environment in the nanotechnology field.

122. Collins DS, Kourtis LC, Thyagarajapuram NR et al. Optimizing the bioavailability of subcutaneously administered biotherapeutics through mechanochemical drivers. Pharm. Res. 34(10), 2000-2011 (2017).

123. Kinnunen HM, Sharma V, Contreras-Rojas LR et al. A novel in vitro method to model the fate of subcutaneously administered biopharmaceuticals and associated formulation components. J. Control. Rel. 214, 94-102 (2015).

124. Richter WF, Jacobsen B. Subcutaneous absorption of biotherapeutics: knowns and unknowns. Drug Metab. Dispos. 42(11), 1890-1905 (2014).

125. Dias C, Abosaleem B, Crispino C, Gao B, Shaywitz A. Tolerability of high-volume subcutaneous injections of a viscous placebo buffer: a randomized, crossover study in healthy subjects. AAPS PharmSciTech 16(5), 1101-1107 (2015).

126. Mathaes R, Koulov A, Joerg S, Mahler HC. Subcutaneous injection volume of biopharmaceuticals—pushing the boundaries. J. Pharm. Sci. 105(8), 2255-2259 (2016).

127. Leveque D. Subcutaneous administration of anticancer agents. Anticancer Res. 34(4), 1579-1586 (2014).

128. Clinical trials. Octreotide. https://clinicaltrials.gov/ct2/show/NCT02299089

129. US FDA. Eligard. www.accessdata.fda.gov/drugsatfda_docs/nda/2016/021343Orig1s033.pdf

130. Sartor O. Eligard: leuprolide acetate in a novel sustained-release delivery system. Urology 61(Suppl. 2), 25-31 (2003).

131. Yang MX, Shenoy B, Disttler M et al. Crystalline monoclonal antibodies for subcutaneous delivery. Proc. Natl Acad. Sci. USA 100(12), 6934-6939 (2003)

132. Johnson HR, Lenhoff AM. Characterization and suitability of therapeutic antibody dense phases for subcutaneous delivery. Mol. Pharm. 10(10), 3582-3591 (2013).

133. Chan LJ, Bulitta JB, Ascher DB et al. PEGylation does not significantly change the initial intravenous or subcutaneous pharmacokinetics or lymphatic exposure of trastuzumab in rats but increases plasma clearance after subcutaneous administration. Mol. Pharm. 12(3), 794-809 (2015).

134. Trainer PJ, Drake WiM, Katznelson L et al. Treatment of acromegaly with the growth hormone recpetor antagonist pagvisomant. $N$. Engl. J. Med. 342, 1171-1177 (2000).

135. Wolin EM, Manon A, Chassaing C et al. Lanreotide depot: an antineoplastic treatment of carcinoid or neuroendocrine tumors. J. Gastrointest. Cancer 47(4), 366-374 (2016).

-• Demonstrates a highly concentrated subcutaneous formulation by simple peptide autoassembly, enabling the prolonged release of the drug.

136. Urquhart J, Fara JW, Willis KL. Rate-controlled delivery systems in drug and hormone research. Annu. Rev. Pharmacol. Toxicol. 24(1), 199-236 (1984).

137. Redding TW, Schally AV, Tice TR, Meyers WE. Long-acting delivery systems for peptides: inhibition of rat prostate tumors by controlled release of [D-Trp6]luteinizing hormone-releasing hormone from injectable microcapsules. Proc. Natl Acad. Sci. USA 81(18 I), 5845-5848 (1984).

138. Hirota K, Doty AC, Ackermann R et al. Characterizing release mechanisms of leuprolide acetate-loaded PLGA microspheres for IVIVC development I: in vitro evaluation. J. Control. Rel. 244, 302-313 (2016).

139. Zhu S, Li X, Lansakara-P DSP, Kumar A, Cui Z. A nanoparticle depot formulation of 4-(N)-stearoyl gemcitabine shows a strong anti-tumour activity. J. Pharm. Pharmacol. 65(2), 236-242 (2013).

140. Sprogøe K, Rau H. TransCon Technology - Ascendis Pharma. https://ascendispharma.com/wp-content/uploads/2018-09-24-Boulder-Presentation-Final.pdf

141. Chatelain P, Malievskiy O, Radziuk K et al. Randomized Phase II study of long-acting transcon GH vs daily GH in childhood GH deficiency. J. Clin. Endocrinol. Metab. 102(5), 1673-1682 (2017).

142. Barz M, Luxenhofer R, Zentel R, Vicent MJ. Overcoming the PEG-addiction: well-defined alternatives to PEG, from structure-property relationships to better defined therapeutics. Polym. Chem. 2(9), 1900-1918 (2011). 
143. Podust VN, Balan S, Sim BC et al. Extension of in vivo half-life of biologically active molecules by XTEN protein polymers. J. Control. Rel. 240, 52-66 (2016).

144. Moreadith RW, Viegas TX, Bentley MD et al. Clinical development of a poly(2-oxazoline) (POZ) polymer therapeutic for the treatment of Parkinson's disease - proof of concept of $\mathrm{POZ}$ as a versatile polymer platform for drug development in multiple therapeutic indications. Eur. Polym. J. 88, 524-552 (2017).

145. Harris JM, Bentley MD, Moreadith RW et al. Tuning drug release from polyoxazoline-drug conjugates. Eur. Polym. J. 120, 109241 (2019).

146. Ahmed KK, Tamer MA, Ghareeb MM, Salem AK. Recent advances in polymeric implants. AAPS PharmSciTech 20(300), (2019).

147. US FDA. Zoladex. https://www.accessdata.fda.gov/drugsatfda_docs/label/2009/019726s050s051s052lbl.pdf

148. US FDA. Lupron. https://www.accessdata.fda.gov/drugsatfda_docs/label/2014/020517s036_019732s041lbl.pdf

149. Sutradhar KB, Sumi CD. Implantable microchip: the futuristic controlled drug delivery system. Drug Deliv. 23(1), 1-11 (2016).

150. Lee SH, Kim BH, Park CG, Lee C, Lim BY, Choy YB. Implantable small device enabled with magnetic actuation for on-demand and pulsatile drug delivery. J. Control. Rel. 286, 224-230 (2018).

- Describes the possibility to deliver drugs on-demand using implantable device, very promising and innovative strategy.

151. Prescott JH, Lipka S, Baldwin S et al. Chronic, programmed polypeptide delivery from an implanted, multireservoir microchip device. Nat. Biotechnol. 24(4), 437-438 (2006).

152. Farra R, Sheppard NF, McCabe L et al. First-in-human testing of a wirelessly controlled drug delivery microchip. Sci. Transl. Med. 4(122), 122ra21-122ra21 (2012).

153. Sinha VR, Trehan A. Biodegradable microspheres for protein delivery. J. Control. Rel. 90(3), 261-280 (2003).

154. Otterson GA, Villalona-Calero MA, Hicks W et al. Phase I/II study of inhaled doxorubicin combined with platinum-based therapy for advanced non-small cell lung cancer. Clin. Cancer Res. 16(8), 2466-2473 (2010).

155. Wittgen BPH, Kunst PWA, Van Der Born K et al. Phase I study of aerosolized SLIT cisplatin in the treatment of patients with carcinoma of the lung. Clin. Cancer Res. 13(8), 2414-2421 (2007).

156. Zarogoulidis P, Eleftheriadou E, Sapardanis I et al. Feasibility and effectiveness of inhaled carboplatin in NSCLC patients. Invest. New Drugs 30(4), 1628-1640 (2012).

157. Kadota K, Imanaka A, Shimazaki M et al. Effects of inhalation procedure on particle behavior and deposition in the airways analyzed by numerical simulation. J. Taiwan Inst. Chem. Eng. 90, 44-50 (2018).

158. Asgharian B, Miller FJ, Price O et al. Modeling particle deposition in the pig respiratory tract. J. Aerosol Sci. 99, 107-124 (2016).

159. Harush-Frenkel O, Bivas-Benita M, Nassar T et al. A safety and tolerability study of differently-charged nanoparticles for local pulmonary drug delivery. Toxicol. Appl. Pharmacol. 246(1-2), 83-90 (2010).

160. Zhang T, Chen Y, Ge Y, Hu Y, Li M, Jin Y. Inhalation treatment of primary lung cancer using liposomal curcumin dry powder inhalers. Acta Pharm. Sin. B 8(3), 440-448 (2018).

161. Rosière R, Van Woensel M, Gelbcke M et al. New folate-grafted chitosan derivative to improve delivery of paclitaxel-loaded solid lipid nanoparticles for lung tumor therapy by inhalation. Mol. Pharm. 15(3), 899-910 (2018).

162. Miglierini P, Bouchekoua M, Rousseau B, Dam Hieu P, Malhaire JP, Pradier O. Impact of the per-operatory application of GLIADEL wafers (BCNU, carmustine) in combination with temozolomide and radiotherapy in patients with glioblastoma multiforme: efficacy and toxicity. Clin. Neurol. Neurosurg. 114(9), 1222-1225 (2012).

163. Fleming AB, Saltzman WM. Pharmacokinetics of the carmustine implant. Clin. Pharmacokinet. 41(6), 403-419 (2002).

164. Menei P, Capelle L, Guyotat J et al. Local and sustained delivery of 5-fluorouracil from biodegradable microspheres for the radiosensitization of malignant glioma: a randomized Phase II trial. Neurosurgery 56(2), 242-247 (2005).

165. Bobo RH, Laske DW, Akbasak A, Morrison PF, Dedrick RL, Oldfield EH. Convection-enhanced delivery of macromolecules in the brain. Proc. Natl Acad. Sci. USA 91(6), 2076-2080 (1994).

166. Song E, Gaudin A, King AR et al. Surface chemistry governs cellular tropism of nanoparticles in the brain. Nat. Commun. 8(May), 1-14 (2017).

167. Conry RM, LoBuglio AF, Wright M et al. Characterization of a messenger RNA polynucleotide vaccine vector. Cancer Res. 55(7), 1397-1400 (1995).

168. Johanning FW, Conry RM, Lobuglio AF et al. A sindbis virus mRNA polynucleotide vector achieves prolonged and high level heterologous gene expression in vivo. Nucleic Acids Res. 23(9), 1495-1501 (1995).

169. Da Silva CG, Camps MGM, Li TMWY, Chan AB, Ossendorp F, Cruz LJ. Co-delivery of immunomodulators in biodegradable nanoparticles improves therapeutic efficacy of cancer vaccines. Biomaterials 220, 119417 (2019).

170. Liang F, Lindgren G, Lin A et al. Efficient targeting and activation of antigen-presenting cells in vivo after modified mRNA vaccine administration in Rhesus Macaques. Mol. Ther. 25(12), 2635-2647 (2017).

171. Clinical trials. mRNA-4157. https://clinicaltrials.gov/ct2/show/NCT03897881?term=mRNA-4157\&rank=1

172. Grabbe S, Haas H, Diken M, Kranz LM, Langguth P, Sahin U. Translating nanoparticulate-personalized cancer vaccines into clinical applications: case study with RNA-lipoplexes for the treatment of melanoma. Nanomedicine 11(20), 2723-2734 (2016). 
173. Kowalski PS, Rudra A, Miao L, Anderson DG. Delivering the messenger: advances in technologies for therapeutic mRNA delivery. Mol. Ther. 27(4), 710-728 (2019). 
